\title{
Accuracy of Computed Tomographic Colonography for the Detection of Polyps and Colorectal Tumors: A Systematic Review and Meta-Analysis
}

\author{
María Chaparro ${ }^{a}$ Javier P. Gisbert ${ }^{a}$ Lourdes del Campo $^{b}$ José Cantero ${ }^{a}$ \\ José Matéa \\ a Department of Gastroenterology and Hepatology, Centro de Investigación Biomédica en Red de Enfermedades \\ Hepáticas y Digestivas, and bepartment of Radiology, La Princesa University Hospital, Madrid, Spain
}

\section{Key Words}

Computed tomography colonography $\cdot$ Virtual colonoscopy $\cdot$ Polyps $\cdot$ Colorectal cancer $\cdot$ Computed tomographic colonoscopy $\cdot$ Computed tomographic colonography

\begin{abstract}
Background: The real accuracy of computed tomographic colonography (CTC) is still unknown. Objective: To perform a meta-analysis of the diagnostic accuracy of CTC for the detection of polyps and colorectal tumors. Methods: Selection of studies: Studies assessing the accuracy of CTC for the detection of colorectal polyps and tumors were selected. Data synthesis: Meta-analyses combining sensitivities, specificities and likelihood ratios (LRs) for the diagnosis of polyps and colorectal tumors were carried out. Results: Forty-seven studies, providing data of 10,546 patients, were included. Overall per-polyp sensitivity of CTC was 66\% (64-68\%), for polyps 6-9 $\mathrm{mm}$ in size it was $59 \%$ (56-61\%), and 76\% (73$79 \%$ ) for polyps larger than $9 \mathrm{~mm}$. Overall per-patient sensitivity was 69\% (66-72\%), for polyps 6-9 mm 60\% (56-65\%), and $83 \%(70-85 \%)$ for lesions larger than $9 \mathrm{~mm}$. Overall CTC specificity was $83 \%$ ( $81-84 \%)$. Positive and negative LRs were $2.9(1.8-4)$ and $0.38(0.27-0.53)$, respectively; for polyps $6-9$ $\mathrm{mm}$ in size, they were $3.8(2.5-5.7)$ and $0.4(0.27-0.59)$, and $12.3(7.7-19.4)$ and $0.19(0.12-0.3)$ for polyps larger than $9 \mathrm{~mm}$.
\end{abstract}

Conclusion: CTC is highly specific for the detection of colorectal polyps and tumors. Some studies reported high sensitivities, but the results of the studies were highly heterogeneous, while the studied variables explained only part of this discrepancy.

Copyright $\odot 2009$ S. Karger AG, Basel

\section{Introduction}

Colorectal cancer (CRC) is the second most common cause of cancer-related death in the western world [1]. The average lifetime incidence of CRC is $6 \%$ and this figure is even higher for those with a family history of colorectal neoplasia with other predisposing conditions [1]. The majority of malignant tumors of colon and rectum arise from preexisting adenomatous polyps [2]. The fact that the progression from adenoma to cancer may take up to 10 years provides the opportunity of early detection of those premalignant lesions, with the use of various screening techniques.

Despite scientific evidence that the screening for CRC can reduce mortality, individuals at risk of developing this disease remain largely underscreened [3]. A major limitation to the widespread implementation of CRC screening is the lack of a single test that meets all the requirements for screening. A diversity of competing and

\section{KARGER}

Fax +4161306 1234 E-Mail karger@karger.ch www.karger.com (c) 2009 S. Karger AG, Basel

0012-2823/09/0801-0001\$26.00/0

Accessible online at:

www.karger.com/dig
María Chaparro Sánchez

Americio 17, PE, $2^{\circ} \mathrm{C}$

ES-28029 Madrid (Spain)

Tel. +34915 202 254, Fax +34913093911

E-Mail mariachs2005@gmail.com 
imperfect tests for CRC screening exists, many of which are required in combination. For many years, barium examination and endoscopy have been the only diagnostic methods for evaluating diseases of the colon [4]. However, in the last 15-20 years, spiral computed tomography (CT) has also been shown to be an essential tool in radiological evaluation of the gastrointestinal tract.

CT colonography (CTC) or virtual colonoscopy was first described in 1994 by Vining et al. [5]. Since then, there have been numerous advances in the CTC technique, which have the potential to improve the accuracy of the test. Modern CT scanners can obtain very thin image slice in a short duration of time with low-radiation exposure [6]. Investigators have developed new methods of stool tagging and electronic colon cleansing to improve the false-negative and positive rates of CTC [7]. Image interpretation can now be enhanced with polyp detection software and three-dimensional (3-D) reconstruction of the bowel lumen, and radiologists are receiving specific training to CTC implementation [8-10].

CTC has been recommended as a less-invasive alternative to conventional colonoscopy (CC) for CRC screening [11]; however, the true accuracy of CTC for the diagnosis of colorectal polyps or tumors is still unknown, as controversial results have been reported by different authors, and the explanation for the heterogeneity of results is insufficiently explained.

The purpose of this study was to perform a systematic review and a meta-analysis of studies evaluating accuracy of CTC compared with CC for detecting colorectal polyps and tumors.

\section{Methods}

\section{Study Identification and Selection}

Bibliographical searches were performed, up to January 2009, in MEDLINE and EMBASE electronic databases, looking for the following words (all fields): 'virtual colonoscopy', 'virtual colonography', 'computed tomography colonography', 'computed tomography colonoscopy', 'CT colonoscopy' or 'CT colonography'. The title and the abstract of potentially relevant studies and review articles were screened for appropriateness before retrieval of the full articles. Inclusion criteria were a prospective, blinded design (in which results of CTC were interpreted independently of findings on colonoscopy or during surgery), enrollment of adult patients who were to undergo CTC after a full bowel preparation, followed by complete colonoscopy or surgery, and use of at least a single detector scanner, with colon insufflation by air or carbon dioxide. In case of multiple articles from the same institution, the dates for study inclusion were evaluated to ensure that there were no overlaps of the patients. Studies that had evaluated computeraided detection systems were excluded.

\section{Study Quality}

The quality of the studies was assessed using the QUADAS ('Quality Assessment of Diagnostic Accuracy Studies') tool [12]. This is the first tool for the assessment of the quality of diagnostic accuracy studies which has been systematically developed and is evidence based [12]. The tool is based on the 14-item questions summarized in table 1, which should each be answered with 'yes', 'no' or 'unclear'. The tool does not incorporate a (global) quality score; the reasons for this are justified in detail in the original paper describing the QUADAS tool [12]. Among these reasons, the most noteworthy is that quality scores ignore the fact that the importance of individual items and the direction of potential biases associated with these items may vary according to the context in which they are applied. Therefore, the application of quality scores, with no consideration of the individual quality items, may dilute or entirely miss potential association.

\section{Data Abstraction}

The following variables were abstracted for the original studies in a predefined data extraction form (table 2): characteristics of the study (year, reference standard, type of contrast used, colonic preparation), patients (number of patients, risk of CRC), scanner (type of viewer, type of contrast, collimation, reconstruction thickness, milliamperes), and study quality. True positives, false positives, false negatives, and true negatives with the CTC were included (tables 3,4 ). If data could not be extracted or calculated from the manuscript with confidence, none were entered.

\section{End Points and Definitions}

The primary end points were the sensitivity and specificity of the assay used. This was either quoted directly in the studies or was extractable from analysis of the true positives, true negatives, false positives, and false negatives on a per-patient and per-polyp basis.

\section{Data Synthesis}

The sensitivity, specificity, positive and negative likelihood ratios (LRs), and their corresponding 95\% confidence intervals were calculated for each study. LRs state how many times more likely particular test results are in patients with disease than in those without disease. LRs can be used to adapt the results of a study to your patients. The LR expresses the relative odds for occurrence of a specific test combination in a patient with polyps as opposed to patients without polyps. Using the Bayes' theorem, the posttest odds that the patient has the disease are estimated by multiplying the pretest odds by the LR. Positive LRs $>10$ and negative LRs $<0.1$ have been noted as providing convincing diagnostic evidence, whereas those $>5$ and $<0.2$ give strong diagnostic evidence [13]. To calculate LRs, if the event of one of the cells of the cross table contained a zero value, 0.5 points were added to all the cells.

The heterogeneity of all indexes was evaluated by the graphic examination of the forest plots, and statistically through a homogeneity test based on the $\chi^{2}$ test. Due to the low power of this test, a minimum cutoff $\mathrm{p}$ value of 0.1 was established as a threshold of homogeneity, lower values indicating heterogeneity. In addition, the $I^{2}$ statistic was calculated to assess the impact of heterogeneity on the results. This statistic which describes the percentage of the variability in effect estimates that it is due to heterogeneity rather than sampling error (chance). A value $>50 \%$ may be considered substantial heterogeneity [14]. 
Table 1. QUADAS ('Quality Assessment of Diagnostic Accuracy Studies') tool

\begin{tabular}{|c|c|c|c|c|c|c|c|c|c|c|c|c|c|c|}
\hline & 1 & 2 & 3 & 4 & 5 & 6 & 7 & 8 & 9 & 10 & 11 & 12 & 13 & 14 \\
\hline Johnson, 2008 [17] & $\mathrm{Y}$ & $\mathrm{Y}$ & $\mathrm{Y}$ & $\mathrm{Y}$ & $\mathrm{Y}$ & $\mathrm{Y}$ & $\mathrm{Y}$ & $\mathrm{Y}$ & $\mathrm{Y}$ & $\mathrm{Y}$ & $\mathrm{Y}$ & $\mathrm{U}$ & $\mathrm{N}$ & $\mathrm{N}$ \\
\hline Kim, 2008 [18] & $\mathrm{Y}$ & $\mathrm{Y}$ & $\mathrm{Y}$ & $\mathrm{Y}$ & $\mathrm{Y}$ & $\mathrm{Y}$ & $\mathrm{Y}$ & $\mathrm{Y}$ & $\mathrm{Y}$ & $\mathrm{Y}$ & $\mathrm{Y}$ & $\mathrm{U}$ & $\mathrm{N}$ & $\mathrm{Y}$ \\
\hline Özsunar, 2008 [19] & $\mathrm{Y}$ & $\mathrm{Y}$ & $\mathrm{Y}$ & $\mathrm{Y}$ & $\mathrm{Y}$ & $\mathrm{Y}$ & $\mathrm{Y}$ & $\mathrm{Y}$ & $\mathrm{Y}$ & $\mathrm{Y}$ & $\mathrm{Y}$ & $\mathrm{U}$ & $\mathrm{N}$ & $\mathrm{N}$ \\
\hline Roberts-Thomson, 2008 [20] & $\mathrm{Y}$ & $\mathrm{Y}$ & $\mathrm{Y}$ & $\mathrm{Y}$ & $\mathrm{Y}$ & $\mathrm{Y}$ & $\mathrm{Y}$ & $\mathrm{Y}$ & $\mathrm{Y}$ & $\mathrm{Y}$ & $\mathrm{Y}$ & $\mathrm{U}$ & $\mathrm{N}$ & $\mathrm{Y}$ \\
\hline Yun, 2007 [21] & $\mathrm{Y}$ & $\mathrm{Y}$ & $\mathrm{Y}$ & $\mathrm{N}$ & $\mathrm{Y}$ & $\mathrm{N}$ & $\mathrm{Y}$ & $\mathrm{Y}$ & $\mathrm{Y}$ & $\mathrm{Y}$ & $\mathrm{Y}$ & $\mathrm{U}$ & $\mathrm{N}$ & $\mathrm{Y}$ \\
\hline Chaparro, 2007 [22] & $\mathrm{Y}$ & $\mathrm{Y}$ & $\mathrm{Y}$ & $\mathrm{Y}$ & $\mathrm{Y}$ & $\mathrm{Y}$ & $\mathrm{Y}$ & $\mathrm{Y}$ & $\mathrm{Y}$ & $\mathrm{Y}$ & $\mathrm{Y}$ & $\mathrm{Y}$ & $\mathrm{Y}$ & $\mathrm{Y}$ \\
\hline MacCarty, 2006 [23] & $\mathrm{Y}$ & $\mathrm{N}$ & $\mathrm{Y}$ & $\mathrm{Y}$ & $\mathrm{Y}$ & $\mathrm{Y}$ & $\mathrm{Y}$ & $\mathrm{N}$ & $\mathrm{Y}$ & $\mathrm{Y}$ & $\mathrm{Y}$ & $\mathrm{U}$ & $\mathrm{N}$ & $\mathrm{Y}$ \\
\hline Haykir, 2006 [24] & $\mathrm{Y}$ & $\mathrm{Y}$ & $\mathrm{Y}$ & $\mathrm{Y}$ & $\mathrm{Y}$ & $\mathrm{Y}$ & $\mathrm{Y}$ & $\mathrm{N}$ & $\mathrm{Y}$ & $\mathrm{Y}$ & $\mathrm{Y}$ & $\mathrm{U}$ & $\mathrm{N}$ & $\mathrm{N}$ \\
\hline Rockey, 2005 [25] & $\mathrm{Y}$ & $\mathrm{Y}$ & $\mathrm{Y}$ & $\mathrm{Y}$ & $\mathrm{Y}$ & $\mathrm{Y}$ & $\mathrm{Y}$ & $\mathrm{N}$ & $\mathrm{Y}$ & $\mathrm{Y}$ & $\mathrm{Y}$ & $\mathrm{U}$ & $\mathrm{N}$ & $\mathrm{N}$ \\
\hline Chung, 2005 [26] & $\mathrm{Y}$ & $\mathrm{Y}$ & $\mathrm{Y}$ & $\mathrm{Y}$ & $\mathrm{Y}$ & $\mathrm{Y}$ & $\mathrm{Y}$ & $\mathrm{Y}$ & $\mathrm{Y}$ & $\mathrm{Y}$ & $\mathrm{Y}$ & $\mathrm{U}$ & $\mathrm{N}$ & $\mathrm{Y}$ \\
\hline Abdel Razek, 2005 [27] & $\mathrm{Y}$ & $\mathrm{N}$ & $\mathrm{Y}$ & $\mathrm{N}$ & $\mathrm{Y}$ & $\mathrm{Y}$ & $\mathrm{Y}$ & $\mathrm{Y}$ & $\mathrm{Y}$ & $\mathrm{Y}$ & $\mathrm{Y}$ & $\mathrm{U}$ & $\mathrm{N}$ & $\mathrm{N}$ \\
\hline Cotton, $2004[28]$ & $\mathrm{Y}$ & $\mathrm{Y}$ & $\mathrm{Y}$ & $\mathrm{Y}$ & $\mathrm{Y}$ & $\mathrm{Y}$ & $\mathrm{Y}$ & $\mathrm{N}$ & $\mathrm{Y}$ & $\mathrm{Y}$ & $\mathrm{N}$ & $\mathrm{U}$ & $\mathrm{N}$ & $\mathrm{Y}$ \\
\hline Macari, 2004 [29] & $\mathrm{Y}$ & $\mathrm{Y}$ & $\mathrm{Y}$ & $\mathrm{Y}$ & $\mathrm{Y}$ & $\mathrm{Y}$ & $\mathrm{Y}$ & $\mathrm{Y}$ & $\mathrm{Y}$ & $\mathrm{Y}$ & $\mathrm{Y}$ & $\mathrm{N}$ & $\mathrm{N}$ & $\mathrm{Y}$ \\
\hline Van Gelder, 2004 [30] & $\mathrm{Y}$ & $\mathrm{Y}$ & $\mathrm{Y}$ & $\mathrm{Y}$ & $\mathrm{Y}$ & $\mathrm{Y}$ & $\mathrm{Y}$ & $\mathrm{Y}$ & $\mathrm{Y}$ & $\mathrm{Y}$ & $\mathrm{Y}$ & $\mathrm{U}$ & $\mathrm{N}$ & $\mathrm{N}$ \\
\hline Macari, 2004 [31] & $\mathrm{Y}$ & $\mathrm{Y}$ & $\mathrm{Y}$ & $\mathrm{Y}$ & $\mathrm{Y}$ & $\mathrm{Y}$ & $\mathrm{Y}$ & $\mathrm{Y}$ & $\mathrm{Y}$ & $\mathrm{Y}$ & $\mathrm{Y}$ & $\mathrm{U}$ & $\mathrm{N}$ & $\mathrm{Y}$ \\
\hline Hoppe, 2004 [32] & $\mathrm{Y}$ & $\mathrm{Y}$ & $\mathrm{Y}$ & $\mathrm{Y}$ & $\mathrm{Y}$ & $\mathrm{Y}$ & $\mathrm{Y}$ & $\mathrm{Y}$ & $\mathrm{Y}$ & $\mathrm{Y}$ & $\mathrm{N}$ & $\mathrm{U}$ & $\mathrm{N}$ & $\mathrm{N}$ \\
\hline Cohnen, 2004 [33] & $\mathrm{Y}$ & $\mathrm{Y}$ & $\mathrm{Y}$ & $\mathrm{U}$ & $\mathrm{Y}$ & $\mathrm{Y}$ & $\mathrm{Y}$ & $\mathrm{Y}$ & $\mathrm{Y}$ & $\mathrm{Y}$ & $\mathrm{Y}$ & $\mathrm{U}$ & $\mathrm{N}$ & $\mathrm{Y}$ \\
\hline Piñol, 2004 [34] & $\mathrm{N}$ & $\mathrm{Y}$ & $\mathrm{Y}$ & $\mathrm{Y}$ & $\mathrm{Y}$ & $\mathrm{Y}$ & $\mathrm{Y}$ & $\mathrm{Y}$ & $\mathrm{Y}$ & $\mathrm{Y}$ & $\mathrm{Y}$ & $\mathrm{U}$ & $\mathrm{N}$ & $\mathrm{N}$ \\
\hline Pickhardt, 2003 [35] & $\mathrm{Y}$ & $\mathrm{Y}$ & $\mathrm{Y}$ & $\mathrm{Y}$ & $\mathrm{Y}$ & $\mathrm{Y}$ & $\mathrm{Y}$ & $\mathrm{Y}$ & $\mathrm{Y}$ & $\mathrm{Y}$ & $\mathrm{N}$ & $\mathrm{U}$ & $\mathrm{N}$ & $\mathrm{N}$ \\
\hline Iannaccone, 2003 [36] & $\mathrm{Y}$ & $\mathrm{Y}$ & $\mathrm{Y}$ & $\mathrm{Y}$ & $\mathrm{Y}$ & $\mathrm{Y}$ & $\mathrm{Y}$ & $\mathrm{Y}$ & $\mathrm{Y}$ & $\mathrm{Y}$ & $\mathrm{Y}$ & $\mathrm{U}$ & $\mathrm{N}$ & $\mathrm{Y}$ \\
\hline Johnson, 2003 [37] & $\mathrm{Y}$ & $\mathrm{Y}$ & $\mathrm{Y}$ & $\mathrm{Y}$ & $\mathrm{Y}$ & $\mathrm{Y}$ & $\mathrm{Y}$ & $\mathrm{Y}$ & $\mathrm{Y}$ & $\mathrm{Y}$ & $\mathrm{N}$ & $\mathrm{U}$ & $\mathrm{N}$ & $\mathrm{N}$ \\
\hline Pineau, 2003 [38] & $\mathrm{Y}$ & $\mathrm{Y}$ & $\mathrm{Y}$ & $\mathrm{Y}$ & $\mathrm{Y}$ & $\mathrm{Y}$ & $\mathrm{Y}$ & $\mathrm{Y}$ & $\mathrm{Y}$ & $\mathrm{Y}$ & $\mathrm{N}$ & $\mathrm{N}$ & $\mathrm{N}$ & $\mathrm{N}$ \\
\hline Taylor, 2003 [39] & $\mathrm{Y}$ & $\mathrm{Y}$ & $\mathrm{Y}$ & $\mathrm{Y}$ & $\mathrm{Y}$ & $\mathrm{Y}$ & $\mathrm{Y}$ & $\mathrm{Y}$ & $\mathrm{Y}$ & $\mathrm{Y}$ & $\mathrm{Y}$ & $\mathrm{U}$ & $\mathrm{N}$ & $\mathrm{N}$ \\
\hline Ginnerup Pedersen, 2003 [40] & $\mathrm{N}$ & $\mathrm{Y}$ & $\mathrm{Y}$ & $\mathrm{Y}$ & $\mathrm{Y}$ & $\mathrm{Y}$ & $\mathrm{Y}$ & $\mathrm{Y}$ & $\mathrm{Y}$ & $\mathrm{U}$ & $\mathrm{U}$ & $\mathrm{U}$ & $\mathrm{N}$ & $\mathrm{N}$ \\
\hline Yee, $2003[41]$ & $\mathrm{Y}$ & $\mathrm{N}$ & $\mathrm{Y}$ & $\mathrm{Y}$ & $\mathrm{Y}$ & $\mathrm{Y}$ & $\mathrm{Y}$ & $\mathrm{Y}$ & $\mathrm{N}$ & $\mathrm{Y}$ & $\mathrm{U}$ & $\mathrm{U}$ & $\mathrm{N}$ & $\mathrm{Y}$ \\
\hline Munikrishnan, 2003 [42] & $\mathrm{Y}$ & $\mathrm{Y}$ & $\mathrm{Y}$ & $\mathrm{U}$ & $\mathrm{Y}$ & $\mathrm{Y}$ & $\mathrm{Y}$ & $\mathrm{Y}$ & $\mathrm{N}$ & $\mathrm{Y}$ & $\mathrm{U}$ & $\mathrm{U}$ & $\mathrm{N}$ & $\mathrm{N}$ \\
\hline Laghi, $2002[43]$ & $\mathrm{Y}$ & $\mathrm{Y}$ & $\mathrm{Y}$ & $\mathrm{Y}$ & $\mathrm{Y}$ & $\mathrm{Y}$ & $\mathrm{Y}$ & $\mathrm{Y}$ & $\mathrm{Y}$ & $\mathrm{Y}$ & $\mathrm{Y}$ & $\mathrm{U}$ & $\mathrm{N}$ & $\mathrm{Y}$ \\
\hline Gluecker, 2002 [44] & $\mathrm{Y}$ & $\mathrm{Y}$ & $\mathrm{Y}$ & $\mathrm{Y}$ & $\mathrm{Y}$ & $\mathrm{Y}$ & $\mathrm{Y}$ & $\mathrm{Y}$ & $\mathrm{N}$ & $\mathrm{Y}$ & $\mathrm{U}$ & $\mathrm{U}$ & $\mathrm{N}$ & $\mathrm{Y}$ \\
\hline Lefere, 2002 [45] & $\mathrm{Y}$ & $\mathrm{Y}$ & $\mathrm{Y}$ & $\mathrm{Y}$ & $\mathrm{Y}$ & $\mathrm{Y}$ & $\mathrm{Y}$ & $\mathrm{Y}$ & $\mathrm{Y}$ & $\mathrm{Y}$ & $\mathrm{N}$ & $\mathrm{U}$ & $\mathrm{N}$ & $\mathrm{Y}$ \\
\hline Macari, 2002 [46] & $\mathrm{Y}$ & $\mathrm{Y}$ & $\mathrm{Y}$ & $\mathrm{Y}$ & $\mathrm{Y}$ & $\mathrm{Y}$ & $\mathrm{Y}$ & $\mathrm{Y}$ & $\mathrm{Y}$ & $\mathrm{Y}$ & $\mathrm{Y}$ & $\mathrm{U}$ & $\mathrm{N}$ & $\mathrm{Y}$ \\
\hline McFarland, 2002 [47] & $\mathrm{Y}$ & $\mathrm{Y}$ & $\mathrm{Y}$ & $\mathrm{Y}$ & $\mathrm{Y}$ & $\mathrm{Y}$ & $\mathrm{Y}$ & $\mathrm{Y}$ & $\mathrm{Y}$ & $\mathrm{Y}$ & $\mathrm{Y}$ & $\mathrm{U}$ & $\mathrm{N}$ & $\mathrm{N}$ \\
\hline Wong, 2002 [48] & $\mathrm{Y}$ & $\mathrm{Y}$ & $\mathrm{Y}$ & $\mathrm{Y}$ & $\mathrm{Y}$ & $\mathrm{Y}$ & $\mathrm{Y}$ & $\mathrm{Y}$ & $\mathrm{Y}$ & $\mathrm{Y}$ & $\mathrm{Y}$ & $\mathrm{N}$ & $\mathrm{N}$ & $\mathrm{Y}$ \\
\hline Yee, 2001 [49] & $\mathrm{Y}$ & $\mathrm{Y}$ & $\mathrm{Y}$ & $\mathrm{Y}$ & $\mathrm{Y}$ & $\mathrm{Y}$ & $\mathrm{Y}$ & $\mathrm{Y}$ & $\mathrm{Y}$ & $\mathrm{Y}$ & $\mathrm{Y}$ & $\mathrm{U}$ & $\mathrm{N}$ & $\mathrm{Y}$ \\
\hline Hara, $2001[50]$ & $\mathrm{Y}$ & $\mathrm{Y}$ & $\mathrm{Y}$ & $\mathrm{Y}$ & $\mathrm{Y}$ & $\mathrm{Y}$ & $\mathrm{Y}$ & $\mathrm{Y}$ & $\mathrm{Y}$ & $\mathrm{Y}$ & $\mathrm{Y}$ & $\mathrm{U}$ & $\mathrm{N}$ & $\mathrm{N}$ \\
\hline Spinzi, 2001 [51] & $\mathrm{Y}$ & $\mathrm{Y}$ & $\mathrm{Y}$ & $\mathrm{Y}$ & $\mathrm{Y}$ & $\mathrm{Y}$ & $\mathrm{Y}$ & $\mathrm{Y}$ & $\mathrm{Y}$ & $\mathrm{Y}$ & $\mathrm{Y}$ & $\mathrm{U}$ & $\mathrm{N}$ & $\mathrm{N}$ \\
\hline Fletcher, 2000 [52] & $\mathrm{Y}$ & $\mathrm{Y}$ & $\mathrm{Y}$ & $\mathrm{Y}$ & $\mathrm{Y}$ & $\mathrm{Y}$ & $\mathrm{Y}$ & $\mathrm{Y}$ & $\mathrm{Y}$ & $\mathrm{Y}$ & $\mathrm{Y}$ & $\mathrm{U}$ & $\mathrm{N}$ & $\mathrm{Y}$ \\
\hline Morrin, $2000[53]$ & $\mathrm{N}$ & $\mathrm{Y}$ & $\mathrm{Y}$ & $\mathrm{Y}$ & $\mathrm{Y}$ & $\mathrm{N}$ & $\mathrm{Y}$ & $\mathrm{Y}$ & $\mathrm{N}$ & $\mathrm{Y}$ & $\mathrm{Y}$ & $\mathrm{U}$ & $\mathrm{N}$ & $\mathrm{N}$ \\
\hline Mendelson, 2000 [54] & $\mathrm{Y}$ & $\mathrm{Y}$ & $\mathrm{Y}$ & $\mathrm{Y}$ & $\mathrm{Y}$ & $\mathrm{Y}$ & $\mathrm{Y}$ & $\mathrm{Y}$ & $\mathrm{Y}$ & $\mathrm{Y}$ & $\mathrm{Y}$ & $\mathrm{U}$ & $\mathrm{N}$ & $\mathrm{N}$ \\
\hline Macari, 2000 [55] & $\mathrm{Y}$ & $\mathrm{Y}$ & $\mathrm{Y}$ & $\mathrm{Y}$ & $\mathrm{Y}$ & $\mathrm{Y}$ & $\mathrm{Y}$ & $\mathrm{Y}$ & $\mathrm{Y}$ & $\mathrm{Y}$ & $\mathrm{Y}$ & $\mathrm{U}$ & $\mathrm{N}$ & $\mathrm{Y}$ \\
\hline Morrin, 2000 [56] & $\mathrm{N}$ & $\mathrm{Y}$ & $\mathrm{Y}$ & $\mathrm{Y}$ & $\mathrm{Y}$ & $\mathrm{Y}$ & $\mathrm{Y}$ & $\mathrm{Y}$ & $\mathrm{N}$ & $\mathrm{U}$ & $\mathrm{N}$ & $\mathrm{U}$ & $\mathrm{N}$ & $\mathrm{N}$ \\
\hline Kay, 2000 [57] & $\mathrm{Y}$ & $\mathrm{Y}$ & $\mathrm{Y}$ & $\mathrm{Y}$ & $\mathrm{Y}$ & $\mathrm{Y}$ & $\mathrm{Y}$ & $\mathrm{Y}$ & $\mathrm{Y}$ & $\mathrm{Y}$ & $\mathrm{Y}$ & $\mathrm{U}$ & $\mathrm{N}$ & $\mathrm{N}$ \\
\hline Miao, 2000 [58] & $\mathrm{Y}$ & $\mathrm{Y}$ & $\mathrm{Y}$ & $\mathrm{Y}$ & $\mathrm{Y}$ & $\mathrm{Y}$ & $\mathrm{Y}$ & $\mathrm{N}$ & $\mathrm{Y}$ & $\mathrm{Y}$ & $\mathrm{Y}$ & $\mathrm{U}$ & $\mathrm{N}$ & $\mathrm{N}$ \\
\hline Fenlon, 1999 [59] & $\mathrm{Y}$ & $\mathrm{Y}$ & $\mathrm{Y}$ & $\mathrm{Y}$ & $\mathrm{Y}$ & $\mathrm{Y}$ & $\mathrm{Y}$ & $\mathrm{Y}$ & $\mathrm{Y}$ & $\mathrm{Y}$ & $\mathrm{Y}$ & $\mathrm{U}$ & $\mathrm{N}$ & $\mathrm{Y}$ \\
\hline Rex, $1999[60]$ & $\mathrm{Y}$ & $\mathrm{Y}$ & $\mathrm{Y}$ & $\mathrm{Y}$ & $\mathrm{Y}$ & $\mathrm{Y}$ & $\mathrm{Y}$ & $\mathrm{Y}$ & $\mathrm{Y}$ & $\mathrm{U}$ & $\mathrm{U}$ & $\mathrm{U}$ & $\mathrm{N}$ & $\mathrm{N}$ \\
\hline Dachman, 1998 [61] & $\mathrm{Y}$ & $\mathrm{Y}$ & $\mathrm{Y}$ & $\mathrm{Y}$ & $\mathrm{Y}$ & $\mathrm{Y}$ & $\mathrm{Y}$ & $\mathrm{Y}$ & $\mathrm{Y}$ & $\mathrm{Y}$ & $\mathrm{Y}$ & $\mathrm{N}$ & $\mathrm{N}$ & $\mathrm{N}$ \\
\hline Royster, 1997 [62] & $\mathrm{N}$ & $\mathrm{Y}$ & $\mathrm{Y}$ & $\mathrm{Y}$ & $\mathrm{Y}$ & $\mathrm{N}$ & $\mathrm{Y}$ & $\mathrm{Y}$ & $\mathrm{Y}$ & $\mathrm{Y}$ & $\mathrm{Y}$ & $\mathrm{U}$ & $\mathrm{N}$ & $\mathrm{N}$ \\
\hline Hara, 1997 [63] & $\mathrm{N}$ & $\mathrm{Y}$ & $\mathrm{Y}$ & $\mathrm{Y}$ & $\mathrm{Y}$ & $\mathrm{Y}$ & $\mathrm{Y}$ & $\mathrm{Y}$ & $\mathrm{Y}$ & $\mathrm{Y}$ & $\mathrm{N}$ & $\mathrm{U}$ & $\mathrm{N}$ & $\mathrm{N}$ \\
\hline
\end{tabular}

$\mathrm{Y}=$ Yes; $\mathrm{N}=$ no; $\mathrm{U}=$ unknown. (1) Was the spectrum of patients representative of the patients who will receive the test in practice? (2) Were selection criteria clearly described? (3) Is the reference standard likely to classify the target condition correctly? (4) Is the period between reference standard and index test short enough to be reasonably sure that the target condition did not change between the two tests? (5) Did the whole sample or a random selection of the sample receive verification using a reference standard? (6) Did patients receive the same reference standard regardless of the index test result? (7) Was the reference standard independent of the index test (i.e. the index test did not form part of the reference standard)?
(8) Was the execution of the index test described in sufficient detail to permit replication of the test? (9) Was the execution of the reference standard described in sufficient detail to permit its replication? (10) Were the index test results interpreted without knowledge of the results of the reference standard? (11) Were the reference standard results interpreted without knowledge of the results of the index test? (12) Were the same clinical data available when test results were interpreted as would be available when the test is used in practice? (13) Were uninterpretable/intermediate test results reported? (14) Were withdrawals from the study explained? 
Table 2. Characteristics of the 47 included studies

\begin{tabular}{|c|c|c|c|c|c|c|c|c|c|c|}
\hline Study, year & $\begin{array}{l}\mathrm{Pa}- \\
\text { tients }\end{array}$ & $\begin{array}{l}\text { Prepara- } \\
\text { tion }\end{array}$ & $\begin{array}{l}\text { Gold } \\
\text { standard }\end{array}$ & $\begin{array}{l}\text { Type of } \\
\text { scanner }\end{array}$ & $\begin{array}{l}\text { Disease } \\
\text { prevalence }\end{array}$ & $\begin{array}{l}\text { Radia- } \\
\text { tion } \\
\text { mAs }\end{array}$ & $\begin{array}{l}\text { Contrast } \\
\text { material }\end{array}$ & $\begin{array}{l}\text { Recon- } \\
\text { struction } \\
\mathrm{mm}\end{array}$ & $\begin{array}{l}\text { Colli- } \\
\text { mation } \\
\mathrm{mm}\end{array}$ & $\begin{array}{l}\text { Mode } \\
\text { imaging }\end{array}$ \\
\hline Johnson, 2008 [17] & 2,600 & PEG, SP & $\mathrm{CC}$ & M & average risk & 50 & oral & 0.8 & 1 & \\
\hline Kim, $2008[18]$ & 214 & PEG, SP & $\mathrm{CC}$ & M & average risk & 120 & IV & 1 & 2 & $2 \mathrm{D}$ with $3 \mathrm{Dc}$ \\
\hline Özsunar, 2008 [19] & 48 & SP & $\mathrm{CC}$ & M & high risk & 175 & no & 1.5 & 3 & \\
\hline Roberts-Thomson, 2008 [20] & 202 & SP & $\mathrm{CC}$ & M & high risk & & no & 1 & 2 & $2 \mathrm{D}$ with $3 \mathrm{Dc}$ \\
\hline Yun, $2007[21]$ & 399 & & $\mathrm{CC}$ & M & high risk & 180 & IV & 1 & 1.5 & $3 \mathrm{D}$ \\
\hline Chaparro, 2007 [22] & 50 & PEG & $\mathrm{CC}$ & $\mathrm{M}$ & high risk & 50 & no & 1.25 & 2 & $2 \mathrm{D}$ with $3 \mathrm{Dc}$ \\
\hline MacCarty, 2006 [23] & 75 & PEG & $\mathrm{CC}$ & M & high risk & 80 & no & & 1.25 & $2 \mathrm{D}$ with $3 \mathrm{Dc}$ \\
\hline Haykir, 2006 [24] & 42 & other & $\mathrm{CC}$ & M & high risk & 150 & oral & & 5 & $2 \mathrm{D}$ with $3 \mathrm{Dc}$ \\
\hline Rockey, 2005 [25] & 614 & other & $\mathrm{CC}$ & M & high risk & & no & 1 & 2.5 & $2 \mathrm{D}$ with $3 \mathrm{Dc}$ \\
\hline Chung, 2005 [26] & 51 & PEG & $\mathrm{CC}, \mathrm{Sg}$ & M & high risk & 160 & IV & 1 & 0.75 & $3 \mathrm{D}$ \\
\hline Abdel Razek, 2005 [27] & 32 & other & $\mathrm{CC}$ & M & high risk & 100 & no & 3 & 5 & $3 \mathrm{D}$ \\
\hline Cotton, $2004[28]$ & 600 & other & $\mathrm{CC}$ & S, M & high risk & & no & 1.5 & 5 & $2 \mathrm{D}$ with $3 \mathrm{Dc}$ \\
\hline Macari, 2004 [29] & 186 & PEG, SP & $\mathrm{CC}$ & M & high risk & 50 & no & 1.6 & 1 & $2 \mathrm{D}$ with $3 \mathrm{Dc}$ \\
\hline Van Gelder, 2004 [30] & 249 & PEG & $\mathrm{CC}$ & M & high risk & 50 & no & 1.25 & 2.5 & $3 \mathrm{D}$ \\
\hline Macari, 2004 [31] & 68 & SP & $\mathrm{CC}$ & M & average risk & 50 & no & 1.25 & 1 & $2 \mathrm{D}$ with $3 \mathrm{Dc}$ \\
\hline Hoppe, 2004 [32] & 92 & other & $\mathrm{CC}$ & M & high risk & 200 & IV & 1 & 2 & $2 \mathrm{D}$ with $3 \mathrm{Dc}$ \\
\hline Cohnen, 2004 [33] & 137 & PEG & $\mathrm{CC}$ & M & high risk & 10 & no & 1.25 & 1 & $3 \mathrm{D}$ \\
\hline Piñol, 2004 [34] & 30 & PEG & $\mathrm{CC}$ & M & high risk & 30 & no & 3 & 5 & $2 \mathrm{D}$ with $3 \mathrm{Dc}$ \\
\hline Pickhardt, 2003 [35] & 1,233 & SP & $\mathrm{CC}$ & $\mathrm{M}$ & average risk & 100 & oral & 1 & 1.25 & $3 \mathrm{D}$ \\
\hline Iannaccone, 2003 [36] & 158 & PEG & $\mathrm{CC}$ & $\mathrm{M}$ & high risk & 10 & IV & 1 & 3 & $2 \mathrm{D}$ with $3 \mathrm{Dc}$ \\
\hline Johnson, 2003 [37] & 703 & PEG & $\mathrm{CC}$ & $\mathrm{S}$ & high risk & 80 & no & 3 & 5 & $2 \mathrm{D}$ with $3 \mathrm{Dc}$ \\
\hline Pineau, 2003 [38] & 205 & $\mathrm{SP}$ & $\mathrm{CC}$ & S & high risk & 200 & oral & 1 & 5 & $3 \mathrm{D}$ \\
\hline Taylor, 2003 [39] & 54 & other & $\mathrm{CC}$ & $\mathrm{M}$ & high risk & 150 & IV & 1.5 & 3 & $2 \mathrm{D}$ with $3 \mathrm{Dc}$ \\
\hline Ginnerup Pedersen, 2003 [40] & 144 & SP & $\mathrm{CC}$ & $\mathrm{M}$ & high risk & 70 & no & 1.6 & 3 & $2 \mathrm{D}$ with $3 \mathrm{Dc}$ \\
\hline Yee, $2003[41]$ & 182 & PEG & $\mathrm{CC}$ & S & high risk & 150 & no & 1.5 & 3 & $3 \mathrm{D}$ \\
\hline Munikrishnan, 2003 [42] & 61 & SP & $\mathrm{CC}$ & M & high risk & 120 & IV & 1 & 1 & $2 \mathrm{D}$ with $3 \mathrm{Dc}$ \\
\hline Laghi, 2002 [43] & 165 & PEG & $\mathrm{CC}$ & S, M & high risk & 120 & IV & 2 & 3 & $3 \mathrm{D}$ \\
\hline Gluecker, 2002 [44] & 50 & PEG & $\mathrm{CC}$ & M & high risk & 90 & no & 2 & 5 & $3 \mathrm{D}$ \\
\hline Lefere, 2002 [45] & 100 & other & $\mathrm{CC}$ & S & high risk & & oral & 5 & 3 & $3 \mathrm{D}$ \\
\hline Macari, 2002 [46] & 105 & other & $\mathrm{CC}$ & M & high risk & 50 & no & 1.25 & 1 & $2 \mathrm{D}$ with $3 \mathrm{Dc}$ \\
\hline McFarland, 2002 [47] & 70 & PEG, SP & $\mathrm{CC}$ & S & high risk & 200 & no & 2 & 5 & $2 \mathrm{D}$ with $3 \mathrm{Dc}$ \\
\hline Wong, 2002 [48] & 71 & SP & $\mathrm{CC}$ & M & high risk & 200 & IV & 1.25 & 3.75 & $3 \mathrm{D}$ \\
\hline Yee, 2001 [49] & 300 & PEG & $\mathrm{CC}$ & $\mathrm{S}$ & high risk & 135 & no & 1.5 & 3 & $3 \mathrm{D}$ \\
\hline Hara, 2001 [50] & 237 & PEG & CC & $\mathrm{S}, \mathrm{M}$ & high risk & 60 & no & 3 & 5 & $3 \mathrm{D}$ \\
\hline Spinzi, 2001 [51] & 96 & PEG & CC & $\mathrm{S}$ & high risk & 230 & no & 2.5 & 5 & $2 \mathrm{D}$ with $3 \mathrm{Dc}$ \\
\hline Fletcher, 2000 [52] & 180 & PEG & $\mathrm{CC}$ & S & high risk & 70 & oral & 3 & 5 & $2 \mathrm{D}$ with $3 \mathrm{Dc}$ \\
\hline Morrin, 2000 [53] & 81 & PEG & $\mathrm{CC}$ & $\mathrm{S}, \mathrm{M}$ & high risk & 120 & IV & 1.5 & 3 & $2 \mathrm{D}$ with $3 \mathrm{Dc}$ \\
\hline Mendelson, 2000 [54] & 53 & other & $\mathrm{CC}$ & S & high risk & 70 & no & 2 & 5 & $3 \mathrm{D}$ \\
\hline Macari, $2000[55]$ & 42 & PEG, SP & $\mathrm{CC}, \mathrm{Sg}$ & $\mathrm{S}$ & average risk & 150 & no & 2.5 & 5 & $3 \mathrm{D}$ \\
\hline Morrin, 2000 [56] & 34 & PEG, SP & $\mathrm{CC}$ & S & high risk & 120 & IV & 1.5 & 3 & $2 \mathrm{D}$ with $3 \mathrm{Dc}$ \\
\hline Kay, 2000 [57] & 2 & $\mathrm{SP}$ & $\mathrm{CC}$ & $\mathrm{M}$ & high risk & 200 & no & 2 & 5 & $3 \mathrm{D}$ \\
\hline Miao, 2000 [58] & 201 & PEG & $\mathrm{CC}$ & $\mathrm{M}$ & high risk & & IV & 4 & 8 & $2 \mathrm{D}$ with $3 \mathrm{Dc}$ \\
\hline Fenlon, 1999 [59] & 100 & PEG & $\mathrm{CC}$ & $\mathrm{S}$ & high risk & 110 & no & 2 & 5 & $3 \mathrm{D}$ \\
\hline Rex, 1999 [60] & 46 & PEG & $\mathrm{CC}$ & $\mathrm{S}$ & average risk & 200 & no & 2 & 5 & $3 \mathrm{D}$ \\
\hline Dachman, 1998 [61] & 44 & PEG & $\mathrm{CC}$ & $\mathrm{S}$ & high risk & 100 & no & 2.5 & 5 & $2 \mathrm{D}$ with $3 \mathrm{Dc}$ \\
\hline Royster, 1997 [62] & 20 & PEG & $\mathrm{CC}, \mathrm{Sg}$ & $\mathrm{S}$ & high risk & 110 & no & 2 & 5 & $3 \mathrm{D}$ \\
\hline Hara, 1997 [63] & 70 & PEG & $\mathrm{CC}$ & S & high risk & 140 & no & 3 & 5 & $3 \mathrm{D}$ \\
\hline
\end{tabular}

PEG = Polyethylene glycol; $\mathrm{SP}=$ sodium phosphate; $\mathrm{CC}=$ conventional colonoscopy $\mathrm{Sg}=$ surgery $; \mathrm{M}=$ multislice; $\mathrm{S}=$ single; $\mathrm{IV}=$ intravenous contrast; $3 \mathrm{Dc}=3$-D confirmation. 
Table 3. Test performance of CTC by per-patient analysis

\begin{tabular}{|c|c|c|c|c|c|c|c|c|c|c|c|c|c|c|c|c|c|}
\hline \multirow[t]{2}{*}{ Author, year } & \multirow{2}{*}{$\begin{array}{l}\mathrm{Pa}- \\
\text { tients } \\
\mathrm{n}\end{array}$} & \multicolumn{4}{|c|}{ Detection of polyps } & \multicolumn{4}{|c|}{ Polyps $<6 \mathrm{~mm}$} & \multicolumn{4}{|c|}{ Polyps 6-9 mm } & \multicolumn{4}{|c|}{ Polyps $>9 \mathrm{~mm}$} \\
\hline & & $\mathrm{TP}$ & FP & FN & $\mathrm{TN}$ & $\mathrm{TP}$ & $\mathrm{FP}$ & $\mathrm{FN}$ & $\mathrm{TN}$ & $\mathrm{TP}$ & FP & FN & $\mathrm{TN}$ & $\mathrm{TP}$ & FP & FN & $\mathrm{TN}$ \\
\hline Johnson, 2008 [17] & 2,600 & & & & & & & & & 193 & 248 & 99 & 2,001 & 98 & 340 & 8 & 2,082 \\
\hline Kim, 2008 [18] & 241 & 37 & 21 & 17 & 166 & & & & & 32 & & 12 & & 9 & & 1 & \\
\hline Özsunar, 2008 [19] & 48 & & & & & & & & & & & & & & & & \\
\hline Roberts-Thomson, 2008 [20] & 202 & 43 & 32 & 26 & 101 & & & & & & & & & & & & \\
\hline Yun, $2007[21]$ & 399 & & & & & & & & & & & & & & & & \\
\hline Chaparro, 2007 [22] & 50 & & & & & 1 & 2 & 16 & 32 & 3 & 5 & 1 & 41 & 4 & 0 & 1 & 45 \\
\hline MacCarty, 2006 [23] & 75 & & & & & 48 & 31 & 226 & 295 & & & & & 37 & 22 & 26 & 528 \\
\hline Haykir, 2006 [24] & 42 & & & & & & & & & 59 & & 57 & & & & & \\
\hline Rockey, 2005 [25] & 614 & 85 & 50 & 70 & 409 & & & & & & & & & & & & \\
\hline Chung, 2005 [26] & 51 & & & & & & & & & & & & & 23 & 23 & 19 & 535 \\
\hline Abdel Razek, 2005 [27] & 32 & & & & & & & & & 23 & 36 & 53 & 488 & & & & \\
\hline Cotton, $2004[28]$ & 600 & 41 & 47 & 63 & 449 & & & & & & & & & 26 & 17.5 & 5 & 200 \\
\hline Macari, 2004 [29] & 249 & 35 & 62 & 10 & 142 & & & & & & & & & & 1 & & 64 \\
\hline Van Gelder, 2004 [30] & 186 & & & & & & & & & & & & & 19 & & & 65 \\
\hline Macari, $2004[31]$ & 68 & & & & & & & & & & & & & & & & \\
\hline Hoppe, 2004 [32] & 92 & 26 & 7 & 8 & 51 & & & & & & & & & & & & \\
\hline Cohnen, 2004 [33] & 137 & & 14 & 19 & & & & & & & & & & 45 & 47 & 3 & 1,138 \\
\hline Piñol, 2004 [34] & 30 & & & & & & & & & & & & & & & & \\
\hline Pickhardt, 2003 [35] & 1,233 & 149 & 217 & 19 & 848 & & & & & & & & & 22.5 & 16.5 & 24.5 & 639 \\
\hline Iannaccone, 2003 [36] & 158 & & & & & & & & & 36 & 56 & 33 & 578 & 18 & 10 & 2 & 175 \\
\hline Johnson, 2003 [37] & 703 & & & & & & & & & & & & & 9 & 0 & & 44 \\
\hline Pineau, 2003 [38] & 205 & 38 & 27 & 7 & 133 & & & & & & & & & 22 & & 1 & \\
\hline Taylor, $2003[39]$ & 54 & & & & & & & & & 14 & & 3 & & & & & \\
\hline Ginnerup Pedersen, 2003 [40] & 148 & 30 & & 3 & & & & & & & & & & & & & \\
\hline Yee, $2003[41]$ & 182 & & & & & & & & & & & & & & & & \\
\hline Munikrishnan, 2003 [42] & 80 & & & & & & & & & & & & & & & & \\
\hline Laghi, $2002[43]$ & 165 & & & & & 9 & 3 & 8 & & & & & & 25 & 0 & 0 & 75 \\
\hline Gluecker, 2002 [44] & 50 & 29 & 2 & 0 & 19 & 65 & 8 & 14 & & 21 & 6 & 2 & 71 & & & & \\
\hline Lefere, $2002[45]$ & 100 & & & & & & & & & & & & & 98 & & 14 & \\
\hline Macari, $2002[46]$ & 105 & & & & & & & & & 57 & & & & 7 & 0 & 1 & \\
\hline McFarland, 2002 [47] & 70 & & & & & & & & & 0 & 0 & 2 & & 47 & 2 & 0 & 251 \\
\hline Wong, $2002[48]$ & 71 & 16 & 3 & 11 & & 5 & & 3 & & 40 & 23 & 2 & 235 & 9.5 & 10 & 4.5 & 212 \\
\hline Yee, 2001 [49] & 300 & & & & & & & & & & & & & & & & \\
\hline Hara, 2001 [50] & 237 & & & & & & & & & & & & & 82 & 6 & 14 & 78 \\
\hline Spinzi, 2001 [51] & 96 & & & & & & & & & & & & & 14 & 0 & 2 & 65 \\
\hline Fletcher, 2000 [52] & 180 & 114 & 14 & 16 & 36 & & & & & 16 & 2 & 6 & 57 & & & & \\
\hline Morrin, $2000[53]$ & 81 & & & & & 14 & 16 & 45 & & & & & & & & & \\
\hline Mendelson, $2000[54]$ & 53 & 15 & 2 & 4 & 32 & & & & & & & & & & & & \\
\hline Macari, $2000[55]$ & 42 & & & & & 4 & & 12 & & & & & & & & & \\
\hline Morrin, 2000 [56] & 34 & & & & & & & & & & & & & 6 & 8 & 7 & \\
\hline Kay, 2000 [57] & 38 & & & & & & & & & 3 & 4 & 12 & & 48 & 2 & 2 & 48 \\
\hline Miao, $2000[58]$ & 201 & 14 & 16 & 45 & & 12 & 8 & 17 & 32 & 47 & 4 & 3 & 46 & 8 & 4 & 2 & 32 \\
\hline Fenlon, 1999 [59] & 100 & & & & & & & & & 3 & & 4 & & & & & \\
\hline Rex, 1999 [60] & 46 & 11 & & 6 & & & & & & & & & & & & & \\
\hline Dachman, 1998 [61] & 44 & & & & & & & & & & & & & 9 & 5.5 & 3 & 52.5 \\
\hline Royster, 1997 [62] & 20 & & & & & & & & & 16 & 17 & 8 & 28 & & & & \\
\hline Hara, 1997 [63] & 70 & 16.5 & 17 & 8.5 & 28 & & & & & & & & & & & & \\
\hline
\end{tabular}

$\mathrm{TP}=$ True positive; $\mathrm{FP}=$ false positive; $\mathrm{FN}=$ false negative; $\mathrm{TN}=$ true negative. 
Table 4. Test performance of CTC by per-polyp analysis

\begin{tabular}{|c|c|c|c|c|c|c|c|c|c|c|c|c|c|}
\hline \multirow[t]{2}{*}{ Author, year } & \multirow{2}{*}{$\begin{array}{l}\mathrm{Pa}- \\
\text { tients } \\
\mathrm{n}\end{array}$} & \multicolumn{3}{|c|}{ Detection of polyps } & \multicolumn{3}{|c|}{ Polyps $<6$ mm } & \multicolumn{3}{|c|}{ Polyps 6-9 mm } & \multicolumn{3}{|c|}{ Polyps $>9 \mathrm{~mm}$} \\
\hline & & $\mathrm{TP}$ & FP & $\mathrm{FN}$ & $\mathrm{TP}$ & FP & $\mathrm{FN}$ & $\mathrm{TP}$ & FP & $\mathrm{FN}$ & $\mathrm{TP}$ & FP & FN \\
\hline Johnson, 2008 [17] & 2,600 & & & & & & & & & & & & \\
\hline Kim, $2008[18]$ & 241 & 95 & & 97 & & & & 45 & & 25 & 13 & 1 & 4 \\
\hline Özsunar, 2008 [19] & 48 & 10 & 0 & 0 & & & & & & & & & \\
\hline Roberts-Thomson, 2008 [20] & 202 & 81 & & 81 & & & & & & & & & \\
\hline Yun, $2007[21]$ & 399 & & & & & & & 101 & & 13 & 41 & 1 & 4 \\
\hline Chaparro, 2007 [22] & 50 & & & & 5 & 1 & 29 & 3 & 6 & 1 & 3 & 0 & 1 \\
\hline MacCarty, 2006 [23] & 75 & 12 & & 18 & & & & 58 & & 39 & & & \\
\hline Haykir, 2006 [24] & 42 & & & & 9 & & 69 & 15 & & & 58 & & \\
\hline Rockey, 2005 [25] & 614 & 93 & & 59 & 16 & 33 & 47 & 27 & & 92 & 6 & & 39 \\
\hline Chung, $2005[26]$ & 51 & 21 & & & 61 & 0 & 46 & 12 & 8 & 14 & & & 0 \\
\hline Abdel Razek, 2005 [27] & 32 & 18 & & 2 & 24 & 13 & 19 & 11.5 & & 3.5 & 28 & & \\
\hline Cotton, $2004[28]$ & 600 & 55 & & 118 & & & & 9 & & 8 & 21 & & 26 \\
\hline Macari, 2004 [29] & 186 & 32.5 & & 9.5 & 19 & & 18 & 11 & & 8 & 20 & & 6 \\
\hline Van Gelder, 2004 [30] & 249 & 32 & 11 & 16 & & & & 12 & 8 & 2 & 3 & 3 & 2 \\
\hline Macari, 2004 [31] & 68 & 12 & & 8 & 37 & 58 & 89 & 14 & 15 & 5 & 12 & & 0 \\
\hline Hoppe, 2004 [32] & 92 & 23 & & 13 & & & & 133 & & 26 & 11 & & 5 \\
\hline Cohnen, 2004 [33] & 137 & 84 & & 51 & & & & 20 & & 4 & 14 & 29 & 3 \\
\hline Piñol, 2004 [34] & 30 & 14 & 15 & 5 & & & & 24 & & 27 & 47 & 15 & 5 \\
\hline Pickhardt, 2003 [35] & 1,233 & 180 & & 30 & 8 & 7 & 7 & 36 & 38 & 12 & 13 & & 4 \\
\hline Iannaccone, 2003 [36] & 158 & 33 & & 4 & & & & 3 & & & 19 & & 0 \\
\hline Johnson, 2003 [37] & 703 & 43 & & 49 & 1 & & 40 & 14 & & 5 & 21 & & 22 \\
\hline Pineau, 2003 [38] & 205 & 57 & 51 & 18 & & & & 71 & & 18 & 3 & 13 & 6 \\
\hline Taylor, 2003 [39] & 54 & 6 & & & 11 & & 80 & 5 & & & 24 & & 0 \\
\hline Ginnerup Pedersen, 2003 [40] & 148 & 38 & & 7 & & & & 14 & & 3 & 38 & & 2 \\
\hline Yee, $2003[41]$ & 182 & 109 & & 21 & 27 & 5 & 13 & 5 & & 10 & 12 & & 3 \\
\hline Munikrishnan, 2003 [42] & 80 & 17 & & & 178 & 64 & 123 & 28 & 9 & 3 & 11 & & 0 \\
\hline Laghi, 2002 [43] & 165 & 25 & & 4 & & & & 19 & 3 & 8 & 9 & & \\
\hline Gluecker, 2002 [44] & 50 & 14 & & 12 & & & & 65 & 61 & 115 & 25 & & 2 \\
\hline Lefere, $2002[45]$ & 100 & 53 & 9 & 3 & & & & 0 & 0 & 2 & 12 & 0 & 0 \\
\hline Macari, $2002[46]$ & 105 & 32 & 4 & 9 & & & & 72 & 97 & 16 & 109 & & \\
\hline McFarland, 2002 [47] & 70 & 174 & 96 & 166 & & & & & & & 7 & 35 & 51 \\
\hline Wong, 2002 [48] & 71 & 34 & 5 & 16 & 2 & & 8 & & & & 64 & 0 & 1 \\
\hline Yee, $2001[49]$ & 300 & 136 & 121 & 20 & 5 & & 3 & 67 & & 75 & & 24 & 4 \\
\hline Hara, $2001[50]$ & 237 & & & & 38 & & & 20 & & 11 & 8 & & \\
\hline Spinzi, 2001 [51] & 96 & & & & 9 & 11 & 70 & 4 & & 14 & 91 & & 5 \\
\hline Fletcher, 2000 [52] & 180 & 158 & & 105 & 29 & 9 & 24 & 3 & 2 & 2 & 20 & & 30 \\
\hline Morrin, $2000[53]$ & 81 & 40 & & 13 & 7 & & 56 & & & & 8 & & 2 \\
\hline Mendelson, 2000 [54] & 53 & 12 & 3 & 17 & 2 & 2 & 11 & 5 & & 8 & & 2 & 3 \\
\hline Macari, 2000 [55] & 42 & 4 & 2 & 2 & 3 & & 0 & 4 & 6 & 21 & & & 0 \\
\hline Morrin, 2000 [56] & 34 & & & & 21 & & 58 & 26 & 0 & 3 & 11 & & \\
\hline Kay, 2000 [57] & 38 & & & & & & & 6 & & 8 & 7 & & 1 \\
\hline Miao, $2000[58]$ & 201 & 20 & 25 & 98 & 9 & & 69 & 1 & 0 & 2 & 20 & 8 & 7 \\
\hline Fenlon, 1999 [59] & 100 & 46 & & 5 & 16 & 33 & 47 & 9 & 0 & & 7 & 0 & 2 \\
\hline Rex, $1999[60]$ & 46 & 13 & & 15 & 61 & 0 & 46 & 12 & 31 & 9 & 5 & & 7 \\
\hline Dachman, 1998 [61] & 44 & 6 & & 3 & 24 & 13 & 19 & & & & 2 & 0 & 1 \\
\hline Royster, 1997 [62] & 20 & 11 & & 3 & & & & & & & 10.5 & 0 & 2 \\
\hline Hara, 1997 [63] & 70 & 22.5 & 37.5 & 13.5 & 19 & & 18 & & & & & 6.5 & 4.5 \\
\hline
\end{tabular}

$\mathrm{TP}=$ True positive; $\mathrm{FP}=$ false positive; $\mathrm{FN}=$ false negative. 


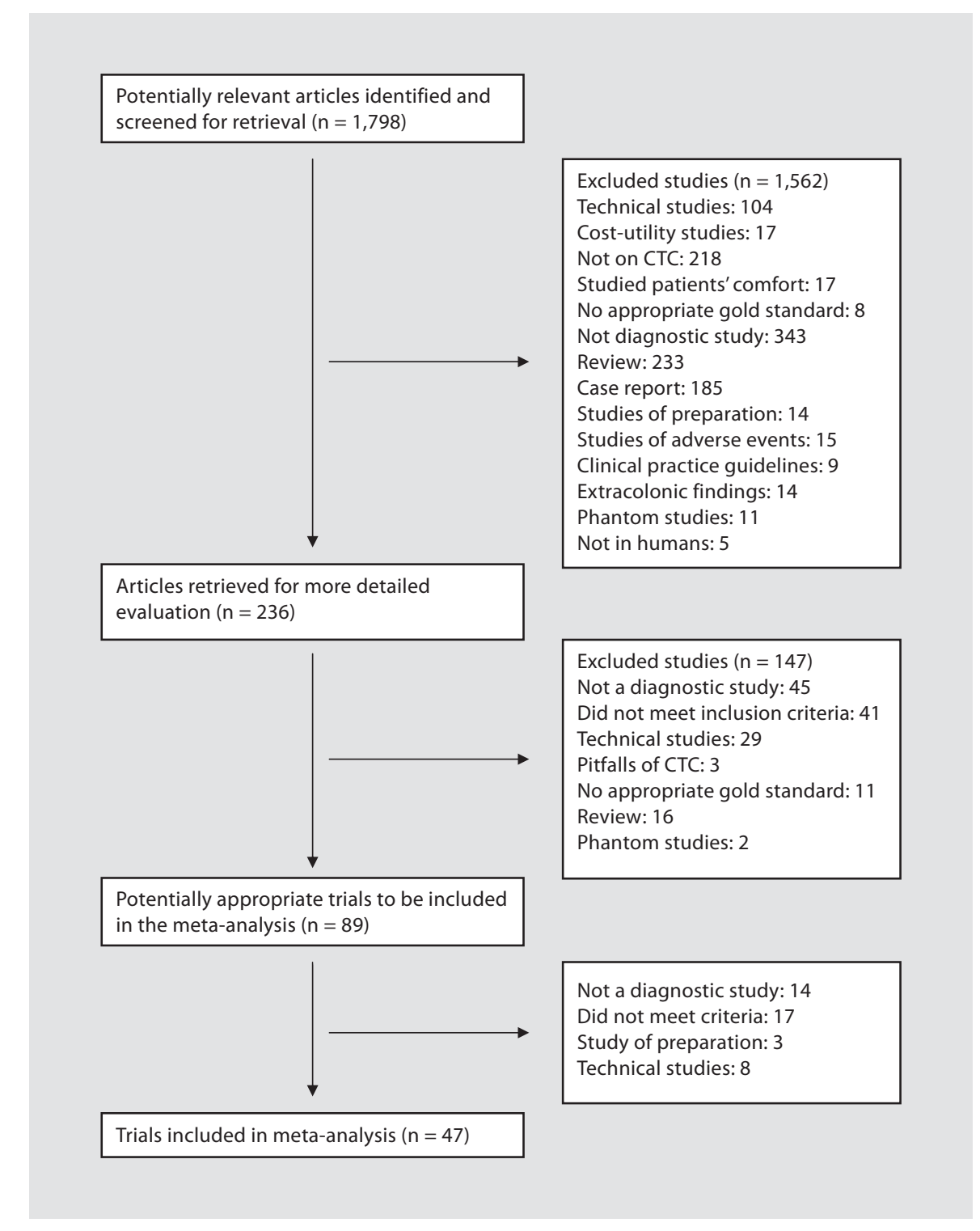

Fig. 1. Flow chart of the literature search and selection process.

Meta-analyses were performed combining the sensitivities, specificities, and LRs of the individual studies in the corresponding pooled indexes. LRs were pooled using a random effects model (DerSimonian and Laird). As a 'threshold effect' was not detected (by the Spearman test and the examination of the plot of sensitivity and specificity on a ROC plane), summary receiver operating characteristic curves were not constructed [15]. Metaanalysis was conducted using Meta-DiSc ${ }^{\odot}$ for Windows Version 1.1.1 software [16].

\section{Subanalyses}

Subgroup analyses were done by polyp size categories (6-9 $\mathrm{mm}$ and larger than $9 \mathrm{~mm}$ ), colonic preparation, use of fecal tagging (yes or no), collimation width and reconstruction interval (in millimeters), type of scanner (single-detector, multidetector or mixed), imaging technique (2-D imaging with $3-\mathrm{D}$ confirmation only when a lesion was observed or always 3-D imaging), radiation dose, and risk of CRC. Analyses for polyps smaller than $6 \mathrm{~mm}$ were not done because the majority of authors did not report 'diminutive' lesions.

\section{Results}

With the aforementioned search strategy, we initially identified 1,798 articles in MEDLINE and EMBASE. The title and abstract of potentially relevant studies and review articles were screened for appropiateness before retrieval of the full articles. Articles that did not meet inclusion criteria were excluded as reported in the flowchart 
Fig. 2. Reported per-polyp sensitivities in

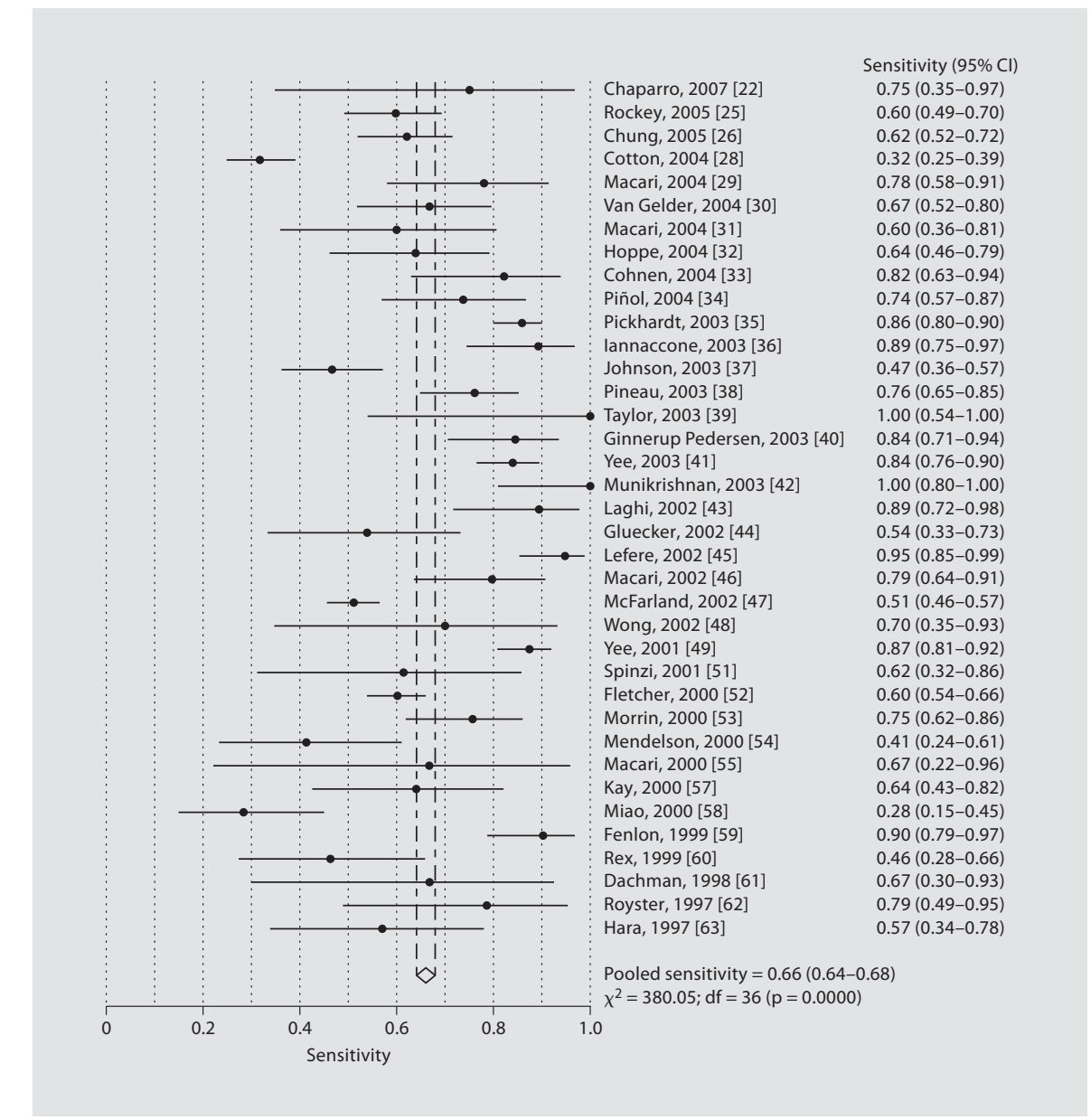
the included studies.

(fig. 1). Finally, 47 studies in total were included in the meta-analysis.

We included 47 prospective studies involving 10,546 patients that compared CTC to the reference standard of CC or surgery [17-63]. Table 2 shows detailed information from individual studies. The average number of participants in a study was 224 . Sixteen studies used singledetector scanners, 27 used mulidetector scanners and 4 used both single and multidetector scanners. CC was the gold standard in 44 studies and surgery was the gold standard in 3 of them. Twenty-four studies used 2-D imaging, with 3-D imaging on selected slices at the discretion of the radiologist; 20 studies used both 2-D and 3-D imaging and 2 studies fly-through imaging with 2-D reconstruction. Ten studies employed sodium phosphate for bowel preparation and 21 employed polyethylene glycol. Six studies used fecal tagging and 12 intravenous contrast. The average collimation was $3.7 \mathrm{~mm}$ and the average reconstruction interval was $2 \mathrm{~mm}$. Six of the studies were done in an average-risk population and the others in a high-risk population. The average radiation intensity employed was $114 \mathrm{~mA}$.

\section{Sensitivity of CTC}

Per-polyp sensitivity for CTC for polyps larger than 6 $\mathrm{mm}$ varied from 28 to $100 \%$. The overall pooled sensitivity for CTC was $66 \%$ [95\% confidence interval (95\% CI): 64-68\%] (fig. 2). The sensitivity increased progressively as polyp size increased: it was $59 \%$ (95\% CI $56-61 \%$; range 16-90\%) for polyps 6-9 mm, and 76\% (95\% CI 73-79\%; range $50-100 \%$ ) for polyps larger than $9 \mathrm{~mm}$ (fig. 3). Each of these analyses was statistically heterogeneous $\left(I^{2}>50 \%\right)$, and most of the variance was attributable to between-study heterogeneity.

Per-patient sensitivity for CTC varied from 24 to $100 \%$ (fig. 4). The overall pooled sensitivity for CTC was 69\% (95\% CI 66-72\%). Sensitivity increased progressively as polyp size increased: it was $60 \%$ (95\% CI 56-65\%) for pa- 


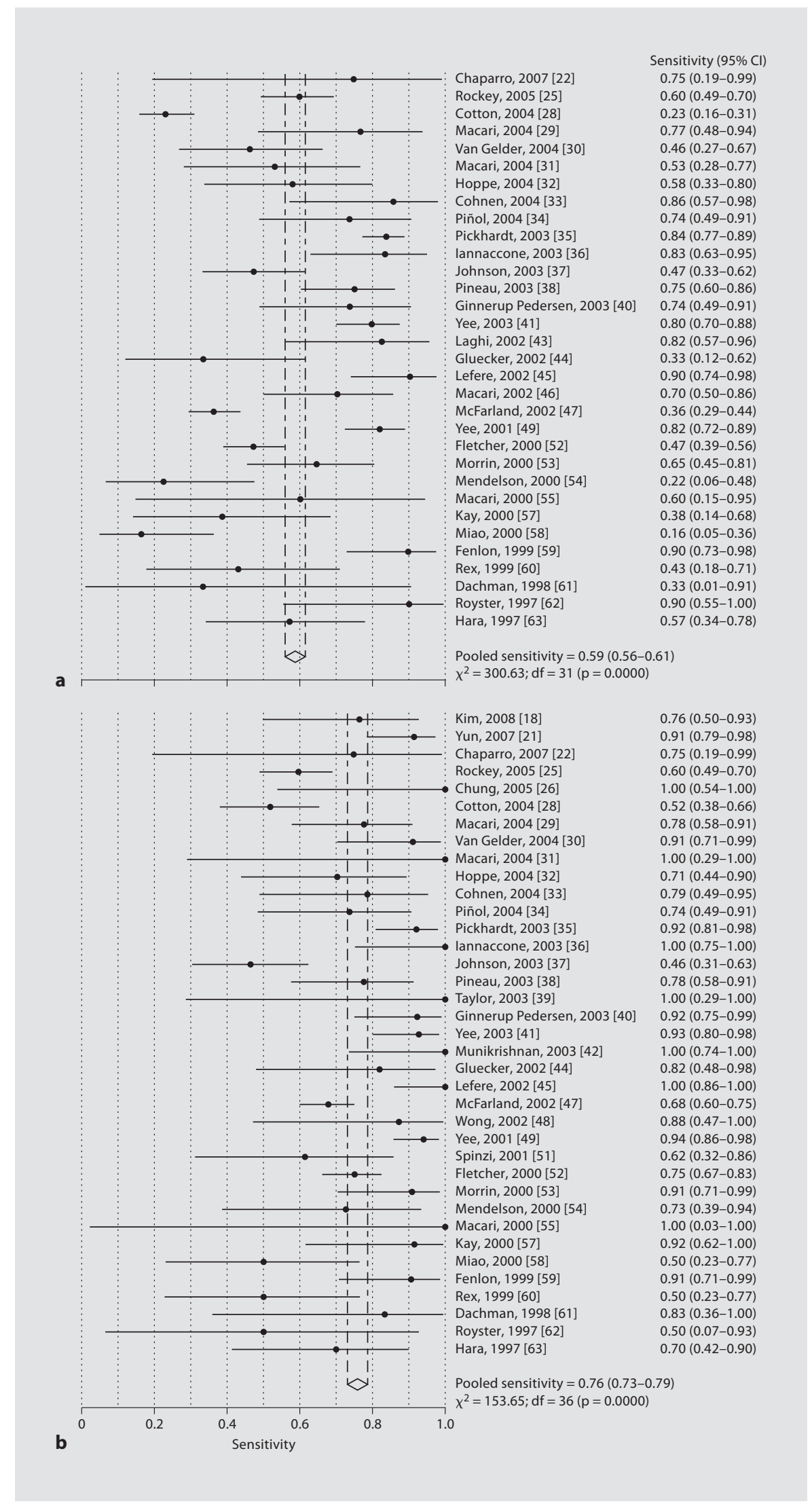

Fig. 3. Reported per-polyp sensitivities in the included studies by polyp size: 6-9 $\mathrm{mm}$ (a) and $>9 \mathrm{~mm}$ (b). 


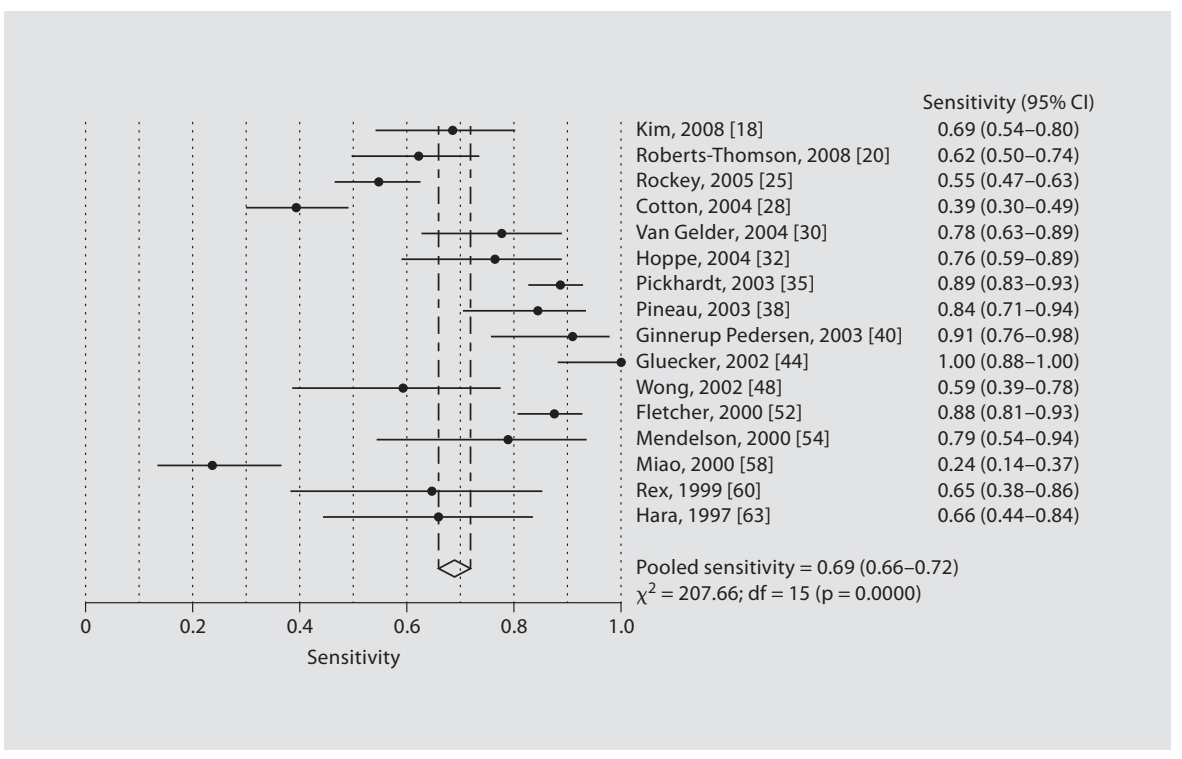

Fig. 4. Reported per-patient sensitivities in the included studies.

tients with polyps of $6-9 \mathrm{~mm}$ (range $20-91 \%$ ), and $83 \%$ (95\% CI 70-85\%; range 46-100\%) for patient with polyps larger than $9 \mathrm{~mm}$ (fig. 5). Each of these analyses was statistically heterogeneous $\left(I^{2}>50 \%\right)$, and most of the variance was attributable to between-study heterogeneity.

\section{Subanalyses}

(1) Use of Phospho-soda for bowel preparation: Mean sensitivity of studies that used Phopho-soda was $83.3 \%$ (95\% CI $79-87 \%)\left(I^{2}=73 \%\right)$. Sensitivity of the 16 studies that did not use Phospho-soda was $62 \%$ (95\% CI 58-66\%) $\left(I^{2}=93 \%\right)$ (fig. 6).

(2) Fecal tagging: Sensitivity of the studies using fecal tagging was $88 \%(95 \%$ CI $84-91 \%)\left(I^{2}<50 \%\right)$. On the other hand, sensitivity of studies without fecal tagging was 59\% (95\% CI 56-63\%) (I $\left.I^{2}=91 \%\right)$ (fig. 7).

(3) Collimation width: Sensitivity of studies that employed a collimation thinner than $5 \mathrm{~mm}$ was $72 \%$ (95\% CI $68-76 \%)\left(I^{2}=89 \%\right)$, while with a collimation of $5 \mathrm{~mm}$ or thicker it was 65\% (95\% CI 60-70\%) $\left(I^{2}=95 \%\right)$ (fig. 8).

(4) Reconstruction thickness: Sensitivity of studies with reconstruction thinner than $3 \mathrm{~mm}$ is $64 \%$ (95\% CI $60-68 \%)\left(I^{2}=90 \%\right)$, while with reconstruction of $3 \mathrm{~mm}$ or thicker it was 58\% (95\% CI 49-67\%) $\left(I^{2}=87 \%\right)$.

(5) Reconstruction mode: Studies that used 2-D imaging with confirmation by 3-D imaging only when considered necessary yielded a sensitivity of $64 \%$ (95\% CI 60 67\%) $\left(I^{2}=90 \%\right)$, whereas studies that always used 3-D imaging had pooled sensitivity of $83 \%$ (95\% CI 78-87\%) $\left(I^{2}=84\right)$.
(6) Radiation: Studies that employed low radiation dose $(<100 \mathrm{~mA})$ reported overall sensitivity of $63 \%(95 \%$ CI $60-67 \%)\left(I^{2}=95\right)$, which is lower than that calculated for studies that used a higher radiation dose (>100 mA) (79\%; 95\% CI 75-83\%) (I $=75 \%)$ (fig. 9).

(7) Population risk: Studies in patients at high risk of polyps or CRC population reported a sensitivity of $65 \%$ (95\% CI $61-68 \%)\left(I^{2}=94 \%\right)$, whereas the sensitivity of studies in average-risk population was $82 \%$ (95\% CI 77$87 \%)\left(I^{2}=83 \%\right)$.

(8) Other variables: We did not find differences in other variables analyzed, including quality of the studies.

\section{Specificity of CTC}

Overall CTC specificity was $83 \%$ (95\% CI $81-84 \%$ ) $\left(I^{2}=89 \%\right)$. Specificity improved as polyp size increased, and the heterogeneity decreased within each stratum. For patients with polyps 6-9 $\mathrm{mm}$ in size, specificity was $90 \%$ (95\% CI $89-91 \%)\left(I^{2}=21 \%\right)$, and increased to $92 \%(95 \%$ CI $92-93 \%)\left(I^{2}=62 \%\right)$ for polyps larger than $9 \mathrm{~mm}$ (fig. 10).

\section{Likelihood Ratios}

Overall positive and negative LRs were 2.9 (1.8-4) and 0.38 (0.27-0.53). Positive and negative LRs for polyps between 6 and $9 \mathrm{~mm}$ were $3.8(2.5-5.7)$ and $0.4(0.27-0.59)$. For polyps larger than $9 \mathrm{~mm}$ in size, positive and negative LRs were 12.3 (7.7-19.4) and 0.19 (0.12-0.3). 
Fig. 5. Reported per-patient sensitivities in the included studies by polyp size: 6-9 $\mathrm{mm}$ (a) and $>9 \mathrm{~mm}$ (b).
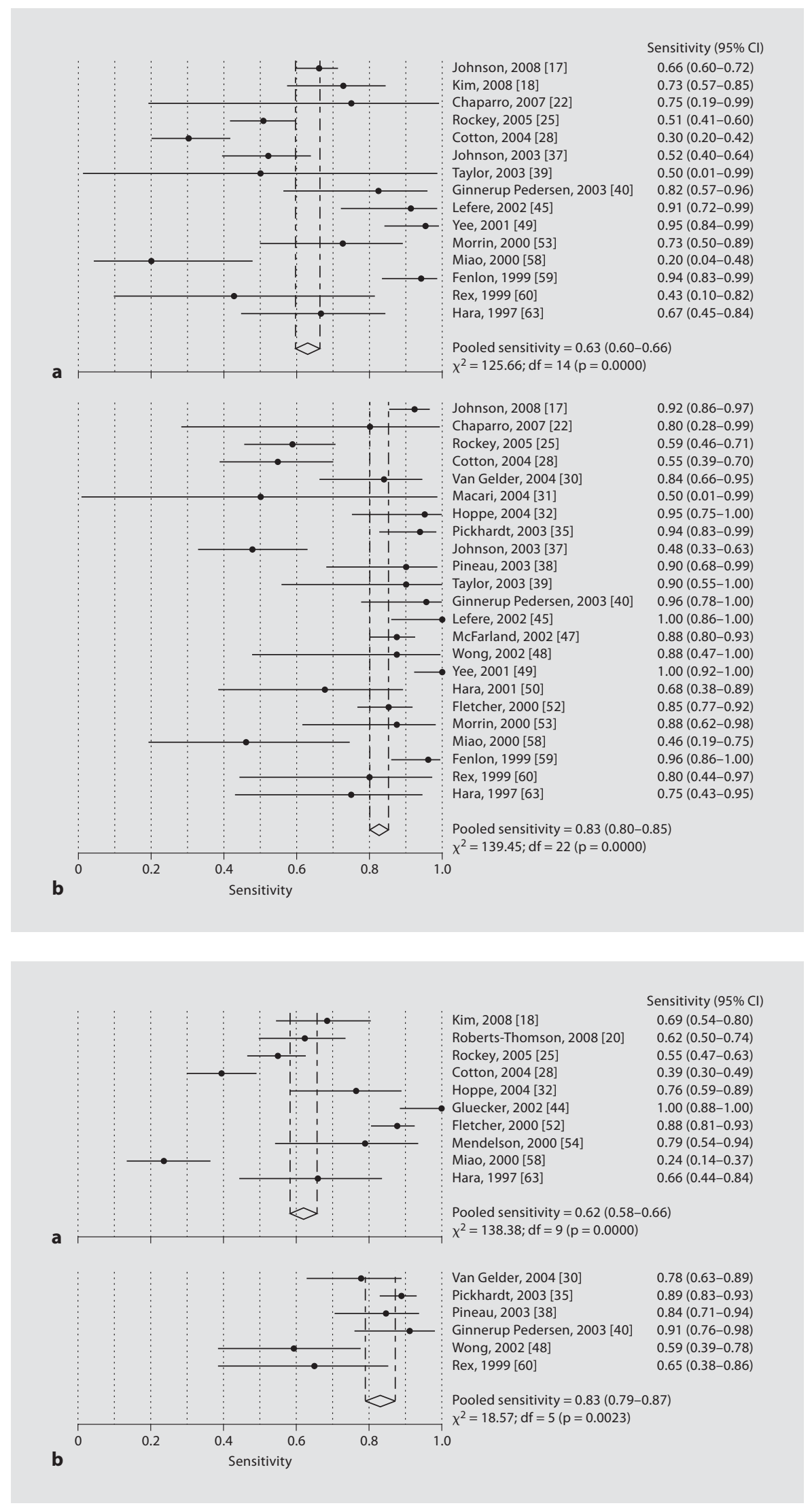

Fig. 6. Reported per-patient sensitivities in the included studies by use of sodium phosphate: without sodium phosphate (a) and with sodium phosphate (b). 
Fig. 7. Reported per-patient sensitivities in the included studies by use of fecal tagging: without fecal tagging (a) and with fecal tagging (b).
Fig. 8. Reported per-patient sensitivities in the included studies by collimation thickness: $>5 \mathrm{~mm}$ (a) and $<5 \mathrm{~mm}$ (b).
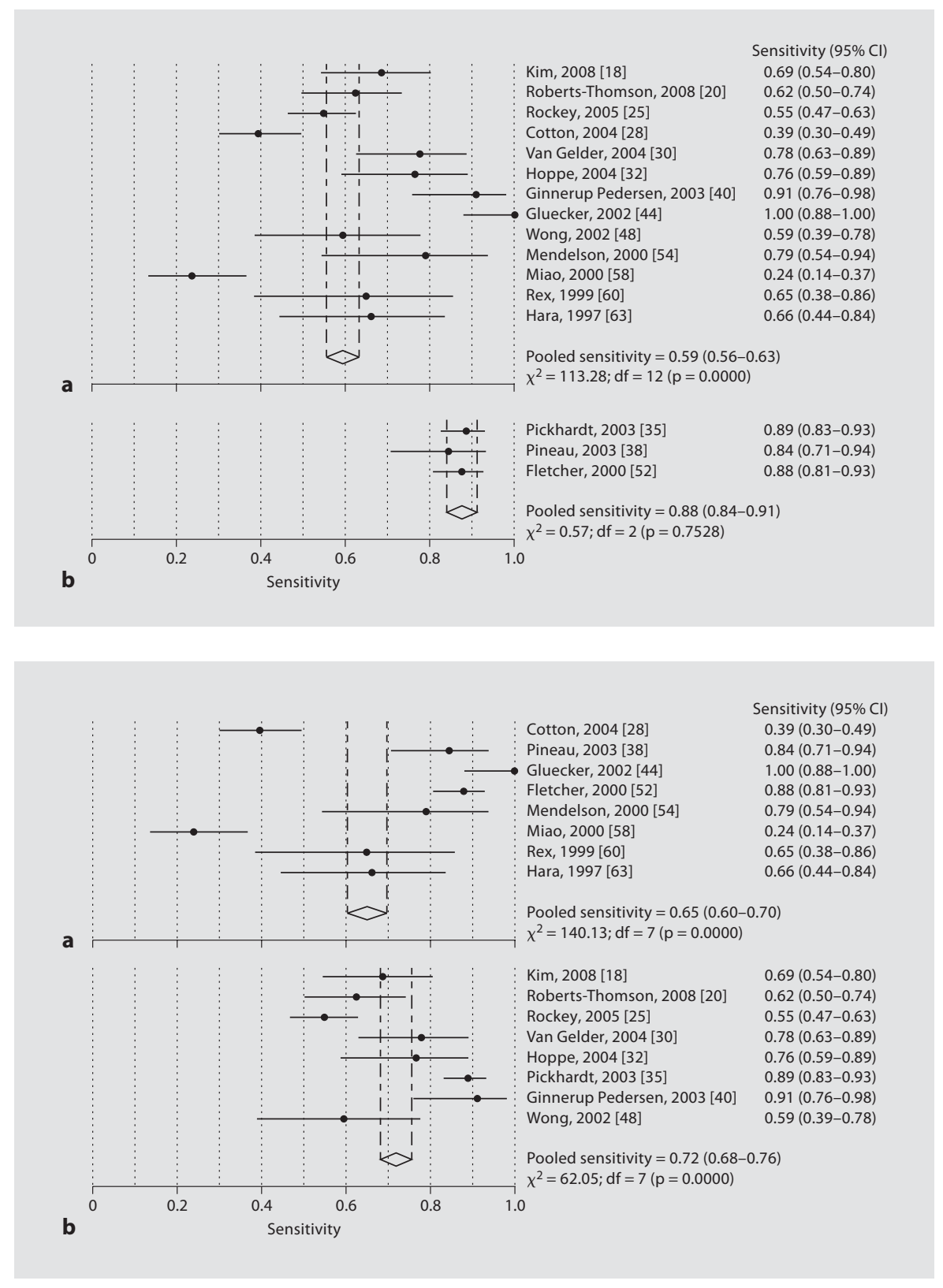

\section{Discussion}

Our meta-analysis showed that CTC is highly specific, but the reported sensitivities for CTC varied widely, even for larger polyps. Any screening method recommended for general use must be demonstrated to be highly and consistently sensitive in a variety of settings [64].

Our analysis revealed some factors that accounted for the wide range of sensitivities. First, scanners that used thinner collimation had higher sensitivity. Second, the mode of imaging also appeared to be important: studies using fly-through technology had higher sensitivity. However, this finding must be interpreted with caution because it was based on data of only two studies. Until the study by Pickhardt et al. [35], all published CTC studies had employed a primary 2-D evaluation of the data, with 3-D endoluminal evaluation limited to problem solving and lesion confirmation. Recent advances in workstation software have transformed 3-D endoluminal navigation of the colon from a cumbersome, time-consuming tech- 
Fig. 9. Reported per-patient sensitivities in the included studies by intensity of radiation: $<100 \mathrm{mAs}$ (a) and >100 mAs (b).
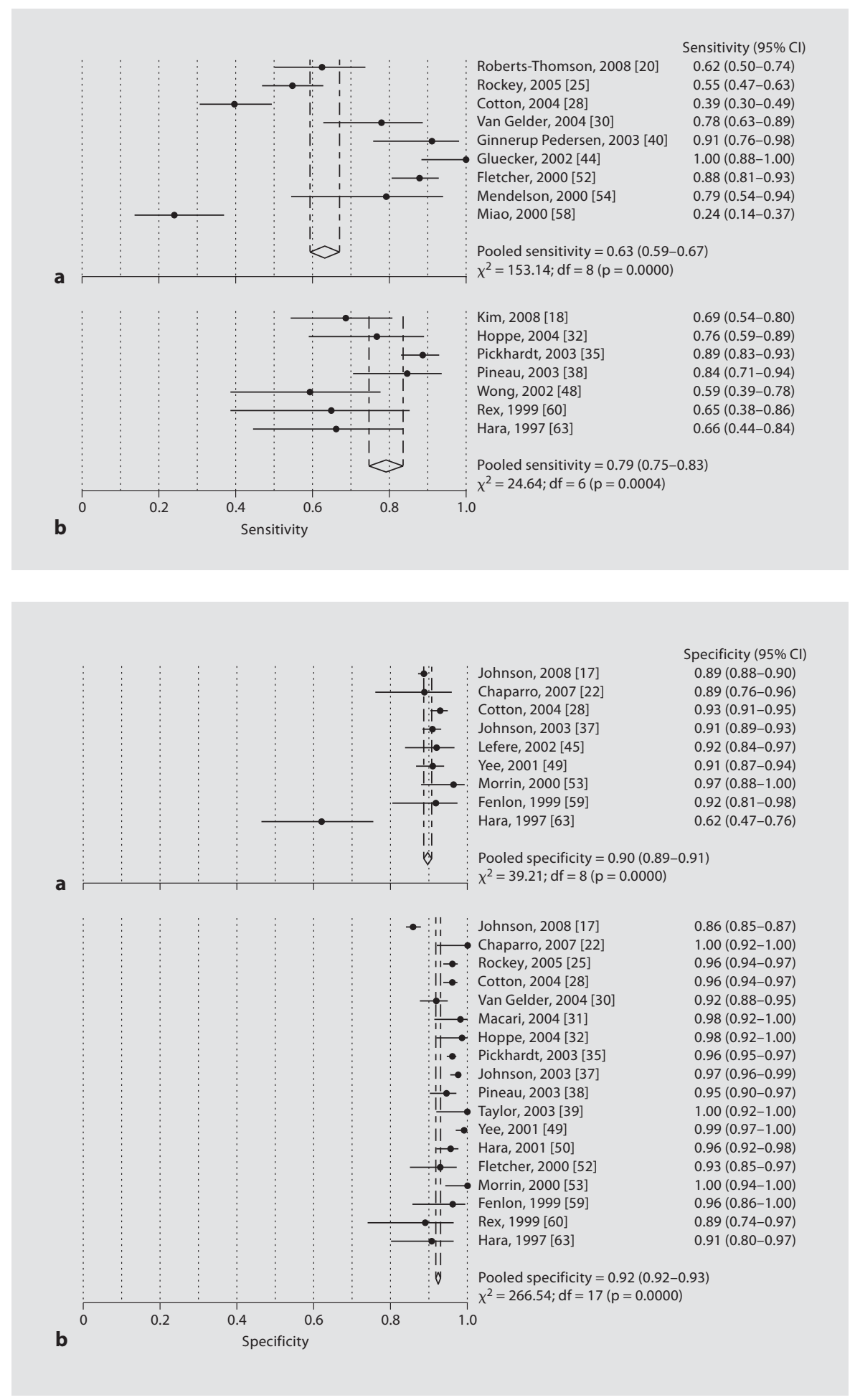

Fig. 10. Reported per-patient specificities in the included studies by polyp size: 6-9 $\mathrm{mm}$ (a) and $>9 \mathrm{~mm}$ (b). 
nique to one that can be performed relatively efficiently. Investigational studies currently in progress are evaluating the relative value of 2-D and 3-D image review [4].

Third, bowel preparation seems to be another important factor influencing accuracy. Studies using sodium phosphate reported better results. In most CTC trials, the investigators have used the bowel preparation usually prescribed by the gastroenterologists. The most common bowel preparations prescribed are polyethylene glycol solution or sodium phosphate plus bisacodyl. With both preparations residual fluid may be left in the colon at the time of examination, but polyethylene glycol solution, in particular, tends to produce a large amount of residual colonic fluid, which can obscure a large position of the colon wall and hide polyps [65]. This problem can be reduced by adding oral iodinated and barium contrast agents to the bowel preparation. We also found higher sensitivities reported in studies using fecal tagging. Additionally, the high-density residual fluid and stool can be electronically removed from the images [66], but this technique is not yet widely available.

Few large and multicenter studies evaluating the accuracy of the CTC have been performed with different results. Recently, the American College of Radiology Imaging Network has carried out a multicenter trial in 2,600 asymptomatic adults, reporting that CTC identified $90 \%$ of polyps larger than $10 \mathrm{~mm}$ [17]. Johnson et al. [37] published a study involving 703 patients, and reported a sensitivity of $46 \%$ for polyps larger than $1 \mathrm{~cm}$ and a specificity from 95 to $98 \%$ for polyps of the same size. Pickhardt et al. [35] published a multicenter trial of 1,233 asymptomatic patients, reporting per-polyp sensitivities for CTC of 85.7, 92.6, and $92.2 \%$ with respect to adenomas of at least $6 \mathrm{~mm}, 8 \mathrm{~mm}$ or at least $10 \mathrm{~mm}$ in diameter; the specificity was $96 \%$. Cotton et al. [28] published a comparative trial of 615 patients, reporting a sensitivity of $55 \%$ for the identification of patients with at least one lesion larger than $10 \mathrm{~mm}$ and a specificity above $90 \%$. Finally, Rockey et al. [25] published a study of 614 symptomatic patients reporting a sensitivity of CTC of $59 \%$ for lesions larger than $10 \mathrm{~mm}$.

Four meta-analyses have been published before ours [64, 67-69]. Mulhall et al. [64] reported sensitivities of 48, 70 and $85 \%$ for polyps smaller than $6 \mathrm{~mm}, 6-9 \mathrm{~mm}$ and larger than $9 \mathrm{~mm}$, respectively. It was also found that sensitivity of CTC was very heterogeneous. The authors found that collimation thickness, mode of imaging and type of detector could explain a small amount of the discrepancy. In that meta-analysis it was concluded that CTC cannot be recommended for general use until the source of heterogeneity is more clearly explained. The last metaanalysis was performed by Rosman and Korsten [69] in 2007. Thirty studies were included, reporting pooled perpatient sensitivities of 82,63 and $56 \%$ for polyps larger than $10 \mathrm{~mm}, 5-10 \mathrm{~mm}$ and smaller than $5 \mathrm{~mm}$, respectively. Our meta-analysis presents some advantages compared to that published previously. First, we included a greater number of studies and a greater number of patients. Second, we performed more detailed subgroup analyses, trying to identify the variables that have more influence on the accuracy of the CTC. The use of fecal tagging, the use of sodium phosphate for bowel cleansing, the thickness of collimation and the mode of imaging seemed to be the most determining variables at the time of obtaining a greater diagnostic accuracy. The radiation dose employed in CTC has been shown to determine some influence in the diagnostic accuracy, although we could not evaluate the effect of the effective dose because we did not have sufficient information for calculating this value. Finally, we calculated LRs that are very important estimators of the accuracy of a diagnostic test. In this respect, we found that CTC provides convincing diagnostic evidence for polyps larger than $9 \mathrm{~mm}$, and gives strong diagnostic evidence for polyps of 6-9 $\mathrm{mm}$ in size.

Overall sensitivity of CTC for detecting polyps smaller than $10 \mathrm{~mm}$ has been poor. However, the importance of these polyps has been debated [70,71]. Since it is not clear whether small polyps should be removed by polypectomy, some radiologists have decided not to report polyps smaller than $5 \mathrm{~mm}$ in size and to recommend CT follow-up of polyps in the 6- to 9 - $\mathrm{mm}$ range by repeating CTC at 1-2 years' intervals [72].

The risks associated with systemic radiation of asymptomatic people remain unknown and are very important in a screening test. A recent report estimated that about $1 \%$ of all cancer deaths in the United States are now related to medical radiation [73]. Extending the risk of radiation to asymptomatic people raises important ethical issues. Some countries do not permit the use of CT scanning in asymptomatic people. The risk of cancer induced by radiation as a result of a CTC study is $0.14 \%$ in a 50 year-old patient [74]. This is similar to the rate of perforation during screening colonoscopies [74], but cancer would be expected to have a higher mortality than colonoscopic perforation. Lower-dose radiation protocols for CTC have been developed. Liedenbaum et al. [75] evaluated the use of effective doses in CTC and they concluded that although the number of CTC protocols with dose modulation had increased substantially since 2004, no significant decrease in effective dose was found. 
CTC had been accepted by institutions for patients with incomplete $\mathrm{CC}$ and for patients that for some reason cannot undergo CC [76], but in previous assessments of the performance of CTC, the American Cancer Society concluded that data were insufficient to recommend screening with CTC for average-risk individuals [77]. Based on the accumulation of evidence since that time, the expert panel has concluded that there are sufficient data now to include CTC as an acceptable option for CRC screening [11].

The implementation of CTC will require quality metrics to be defined and implemented in clinical practice. In 2005, the ACR Practice Guideline for the Performance of Computed Tomography Colonography in Adults was published, encompassing the techniques, quality control, clinical uses, training and communication of results of CTC [78]. An update of these guidelines is planned following publication of the results of the ACRIN CT screening trial [11].

The management of CTC findings is an important part of a CTC screening program. Nowadays the American Cancer Society, the US Multi-Society Task Force on Colorectal Cancer, the American College of Radiology and the ESGAR recommend that all patients with one or more polyps $\geq 10 \mathrm{~mm}$ or 3 or more polyps $\geq 6 \mathrm{~mm}$ should be referred for colonoscopy $[11,79]$. Such polyps must be removed if found at CC because of the risk, albeit low, of advanced neoplasia [80]. Patients with one or two lesions of 6-9 mm should be recommended interval surveillance after up to 3 years depending on several factors [11, 79]. Patients who decline CC or who are not good candidates for CC should be offered surveillance with CTC. At this time, optimal management of patients whose largest polyp is $6 \mathrm{~mm}$ detected on CTC is uncertain, because the risk of advanced features in these polyps is very low (1.7\%) [80]. It is recommended to repeat exams every 5 years if the initial CTC is negative for significant polyps until further studies are completed and are able to provide additional guidelines [11].

In conclusion, CTC offers high sensitivity and specificity for the detection of CRC and polyps larger than 10 $\mathrm{mm}$ in size. It is the method of choice for incomplete colonoscopies and for patients that for some reason cannot undergo CC. The wide range of sensitivities that have been reported and cannot be completely explained must be resolved. The use of CTC in routine screening should be subject to quality control with prescribed examination standards.

\section{Acknowledgment}

CIBEREHD is funded by the Instituto de Salud Carlos III.

\section{References}

1 Xynopoulos D, Stasinopoulou M, Dimitroulopoulos D, Tsamakides K, Arhavlis E, Kontou $\mathrm{M}$, et al: Colorectal polyp detection with virtual colonoscopy (computed tomographic colonography); the reliability of the method. Hepatogastroenterology 2002;49:124127.

-2 Vogelstein B, Fearon ER, Hamilton SR, Kern SE, Preisinger AC, Leppert M, et al: Genetic alterations during colorectal-tumor development. N Engl J Med 1988;319:525-532.

-3 Bowdy M: Lag in colorectal screening rates prompts innovation. J Natl Cancer Inst 1998; 90:886-887.

4 Heiken JP, Peterson CM, Menias CO: Virtual colonoscopy for colorectal cancer screening: current status. Cancer Imaging 2005;5(Spec No A):S133-S139.

5 Vining DJ, Gelfand DW, Bechthold RE, Scharling ES, Grishaw EK, Shifrin RY: Technical feasibility of colon imaging with helical CT and virtual reality. AJR Am J Roentgenol 1994; $162: 104$
-6 Laghi A, Lannaccone R, Panebianco V, Carbone L, Passariello R: Multislice CT colonography: technical developments. Semin Ultrasound CT MR 2001;22:425-431.

7 Thomeer M, Carbone I, Bosmans H, Kiss G, Bielen D, Vanbeckevoort D, et al: Stool tagging applied in thin-slice multidetector computed tomography colonography. J Comput Assist Tomogr 2003;27:132-139.

$>8$ Yoshida H, Dachman AH: Computer-aided diagnosis for CT colonography. Semin Ultrasound CT MR 2004;25:419-431.

-9 Nicholson FB, Barro JL, Bartram CI, Dehmeshki J, Halligan S, Taylor S, et al: The role of CT colonography in colorectal cancer screening. Am J Gastroenterol 2005;100: 2315-2323.

10 Summers RM: Challenges for computer-aided diagnosis for CT colonography. Abdom Imaging 2002;27:268-274.
11 Levin B, Lieberman DA, McFarland B, Andrews KS, Brooks D, Bond J, et al: Screening and surveillance for the early detection of colorectal cancer and adenomatous polyps, 2008: a joint guideline from the American Cancer Society, the US Multi-Society Task Force on Colorectal Cancer, and the American College of Radiology. Gastroenterology 2008;134:1570-1595.

12 Whiting P, Rutjes AW, Reitsma JB, Bossuyt PM, Kleijnen J: The development of QUADAS: a tool for the quality assessment of studies of diagnostic accuracy included in systematic reviews. BMC Med Res Methodol 2003;3:25

13 Jaeschke R, Guyatt G, Sackett DL: Users' guides to the medical literature. III. How to use an article about a diagnostic test. B. What are the results and will they help me in caring for my patients? JAMA 1994;271:703-707.

14 Higgins JP, Thompson SG, Deeks JJ, Altman DG: Measuring inconsistency in meta-analyses. BMJ 2003;327:557-560. 
15 Deeks J: Systematic reviews in health care: systematic reviews of evaluations of diagnostic and screening tests. BMJ 2001;323: 157-162.

16 Zamora J, Abraira V, Muriel A, Khan K, Coomarasamy A: Meta-DiSc: a software for meta-analysis of test accuracy data. BMC Med Res Methodol 2006;6:31.

$\checkmark 17$ Johnson CD, Chen MH, Toledano AY, Heiken JP, Dachman A, Kuo MD, et al: Accuracy of CT colonography for detection of large adenomas and cancers. N Engl J Med 2008; 359:1207-1217.

-18 Kim YS, Kim N, Kim SH, Park MJ, Lim SH, Yim JY, et al: The efficacy of intravenous contrast-enhanced 16-raw multidetector CT colonography for detecting patients with colorectal polyps in an asymptomatic population in Korea. J Clin Gastroenterol 2008; 42:791-798.

19 Özsunar Y, Coskun G, Delibas N, Uz B, Yukselen V: Diagnostic accuracy and tolerability of contrast enhanced CT colonoscopy in symptomatic patients with increased risk for colorectal cancer. Eur J Radiol 2008, e-pub ahead of print. doi:10.1016/j.cjrad. 2008.05.013.

-20 Roberts-Thomson IC, Tucker GR, Hewett PJ, Cheung P, Sebben RA, Khoo EE, et al: Singlecenter study comparing computed tomography colonography with conventional colonoscopy. World J Gastroenterol 2008;14: 469-473.

-21 Yun JY, Ro HJ, Park JB, Choi JB, Chung JE, Kim YJ, et al: Diagnostic performance of CT colonography for the detection of colorectal polyps. Korean J Radiol 2007;8:484-491.

-22 Chaparro Sanchez M, del Campo Val L, Mate Jimenez J, Cantero Perona J, Barbosa A, Olivares $\mathrm{D}$, et al: Computed tomography colonography compared with conventional colonoscopy for the detection of colorectal polyps. Gastroenterol Hepatol 2007;30:375380.

$\checkmark 23$ MacCarty RL, Johnson CD, Fletcher JG, Wilson LA: Occult colorectal polyps on CT colonography: implications for surveillance. AJR Am J Roentgenol 2006;186:1380-1383.

-24 Haykir R, Karakose S, Karabacakoglu A, Sahin M, Kayacetin E: Three-dimensional MR and axial CT colonography versus conventional colonoscopy for detection of colon pathologies. World J Gastroenterol 2006;12: 2345-2350.

-25 Rockey DC, Paulson E, Niedzwiecki D, Davis W, Bosworth $\mathrm{HB}$, Sanders L, et al: Analysis of air contrast barium enema, computed tomographic colonography, and colonoscopy: prospective comparison. Lancet 2005; 365:305-311.

-26 Chung DJ, Huh KC, Choi WJ, Kim JK: CT colonography using 16-MDCT in the evaluation of colorectal cancer. AJR Am J Roentgenol 2005;184:98-103.
27 Abdel Razek AA, Abu Zeid MM, Bilal M, Abdel Wahab NM: Virtual CT colonoscopy versus conventional colonoscopy: a prospective study. Hepatogastroenterology 2005;52: 1698-1702.

28 Cotton PB, Durkalski VL, Pineau BC, Palesch YY, Mauldin PD, Hoffman B, et al: Computed tomographic colonography (virtual colonoscopy): a multicenter comparison with standard colonoscopy for detection of colorectal neoplasia. JAMA 2004;291:17131719.

29 Macari M, Bini EJ, Jacobs SL, Lui YW, Laks S, Milano A, et al: Significance of missed polyps at CT colonography. AJR Am J Roentgenol 2004; 183:127-134.

-30 Van Gelder RE, Nio CY, Florie J, Bartelsman JF, Snel P: Computed tomographic colonography compared with colonoscopy in patients at increased risk of colorectal cancer. Gastroenterology 2004;127:41-48.

-31 Macari M, Bini EJ, Jacobs SL, Naik S, Lui YW, Milano A, et al: Colorectal polyps and cancers in asymptomatic average-risk patients: evaluation with CT colonography. Radiology 2004;230:629-636.

-32 Hoppe H, Quattropani C, Spreng A, Mattich J, Netzer P, Dinkel HP: Virtual colon dissection with CT colonography compared with axial interpretation and conventional colonoscopy: preliminary results. AJR Am J Roentgenol 2004;182:1151-1158.

33 Cohnen M, Vogt C, Beck A, Andersen K, Heinen W, vom Dahl S, et al: Feasibility of MDCT colonography in ultra-low-dose technique in the detection of colorectal lesions: comparison with high-resolution video colonoscopy. AJR Am J Roentgenol 2004; 183:1355-1359.

34 Piñol V, Pagès M, Castells A, Bellot P, Carrión JA, Martín M, Caballería J, Ayuso C, Bordas JM, Piqué JM: Utilidad de la colonografía por tomografía computarizada en la detección de pólipos colorrectales. Med Clin (Barc) 2004;123:41-44.

35 Pickhardt PJ, Choi JR, Hwang I, Butler JA, Puckett ML, Hildebrandt HA, et al: Computed tomographic virtual colonoscopy to screen for colorectal neoplasia in asymptomatic adults. N Engl J Med 2003;349:21912200.

-36 Iannaccone R, Laghi A, Catalano C, Brink JA, Mangiapane F, Trenna S, et al: Detection of colorectal lesions: lower-dose multi-detector row helical CT colonography compared with conventional colonoscopy. Radiology 2003;229:775-781.

37 Johnson CD, Harmsen WS, Wilson LA, Maccarty RL, Welch TJ, Ilstrup DM, et al: Prospective blinded evaluation of computed tomographic colonography for screen detection of colorectal polyps. Gastroenterology 2003; 125:311-319.
38 Pineau BC, Paskett ED, Chen GJ, Espeland MA, Phillips K, Han JP, et al: Virtual colonoscopy using oral contrast compared with colonoscopy for the detection of patients with colorectal polyps. Gastroenterology 2003;125:304-310.

39 Taylor SA, Halligan S, Saunders BP, Morley S, Riesewyk C, Atkin W, et al: Use of multidetector-row CT colonography for detection of colorectal neoplasia in patients referred via the Department of Health '2-Week-wait' initiative. Clin Radiol 2003;58:855-861.

40 Ginnerup Pedersen B, Christiansen TE, Bjerregaard NC, Ljungmann K, Laurberg S: Colonoscopy and multidetector-array computed-tomographic colonography: detection rates and feasibility. Endoscopy 2003;35: 736-742.

41 Yee J, Kumar NN, Hung RK, Akerkar GA, Kumar PR, Wall SD: Comparison of supine and prone scanning separately and in combination at CT colonography. Radiology 2003;226:653-661.

42 Munikrishnan V, Gillams AR, Lees WR, Vaizey CJ, Boulos PB: Prospective study comparing multislice CT colonography with colonoscopy in the detection of colorectal cancer and polyps. Dis Colon Rectum 2003; 46:1384-1390.

43 Laghi A, Iannaccone R, Trenna S, Mangiapane F, Sinibaldi G, Piacentini F, et al: Multislice spiral CT colonography in the evaluation of colorectal neoplasms. Radiol Med (Torino) 2002;104:394-403.

44 Gluecker TM, Fletcher JG: CT colonography (virtual colonoscopy) for the detection of colorectal polyps and neoplasms: current status and future developments. Eur J Cancer 2002;38:2070-2078.

45 Lefere PA, Gryspeerdt SS, Dewyspelaere J, Baekelandt M, Van Holsbeeck BG: Dietary fecal tagging as a cleansing method before CT colonography: initial results polyp detection and patient acceptance. Radiology 2002; 224:393-403

46 Macari M, Bini EJ, Xue X, Milano A, Katz SS, Resnick D, et al: Colorectal neoplasms: prospective comparison of thin-section lowdose multi-detector row CT colonography and conventional colonoscopy for detection. Radiology 2002;224:383-392.

47 McFarland EG, Pilgram TK, Brink JA, McDermott RA, Santillan CV, Brady PW, et al: CT colonography: multiobserver diagnostic performance. Radiology 2002;225:380-390.

-48 Wong BC, Wong WM, Chan JK, Lai KC, Hu WH, Chan CK, et al: Virtual colonoscopy for the detection of colorectal polyps and cancers in a Chinese population. J Gastroenterol Hepatol 2002; 17:1323-1327.

49 Yee J, Akerkar GA, Hung RK, Steinauer-Gebauer AM, Wall SD, McQuaid KR: Colorectal neoplasia: performance characteristics of CT colonography for detection in 300 patients. Radiology 2001;219:685-692. 
-50 Hara AK, Johnson CD, MacCarty RL, Welch TJ, McCollough CH, Harmsen WS: CT colonography: single- versus multi-detector row imaging. Radiology 2001;219:461-465.

-51 Spinzi G, Belloni G, Martegani A, Sangiovanni A, Del Favero C, Minoli G: Computed tomographic colonography and conventional colonoscopy for colon diseases: a prospective, blinded study. Am J Gastroenterol 2001;96:394-400.

- 52 Fletcher JG, Johnson CD, Welch TJ, MacCarty RL, Ahlquist DA, Reed JE, et al: Optimization of CT colonography technique: prospective trial in 180 patients. Radiology 2000;216: 704-711.

53 Morrin MM, Farrell RJ, Raptopoulos V, McGee JB, Bleday R, Kruskal JB: Role of virtual computed tomographic colonography in patients with colorectal cancers and obstructing colorectal lesions. Dis Colon Rectum 2000;43:303-311.

54 Mendelson RM, Foster NM, Edwards JT, Wood CJ, Rosenberg MS, Forbes GM: Virtual colonoscopy compared with conventional colonoscopy: a developing technology. Med J Aust 2000;173:472-475.

-55 Macari M, Milano A, Lavelle M, Berman P, Megibow AJ: Comparison of time-efficient CT colonography with two- and three-dimensional colonic evaluation for detecting colorectal polyps. AJR Am J Roentgenol 2000;174:1543-1549.

56 Morrin MM, Farrell RJ, Kruskal JB, Reynolds K, McGee JB, Raptopoulos V: Utility of intravenously administered contrast material at CT colonography. Radiology 2000; 217:765-771.

-57 Kay CL, Kulling D, Hawes RH, Young JW, Cotton PB: Virtual endoscopy - comparison with colonoscopy in the detection of spaceoccupying lesions of the colon. Endoscopy 2000;32:226-232.

-58 Miao YM, Amin Z, Healy J, Burn P, Murugan $\mathrm{N}$, Westaby $\mathrm{D}$, et al: A prospective single centre study comparing computed tomography pneumocolon against colonoscopy in the detection of colorectal neoplasms. Gut 2000;47:832-837.

-59 Fenlon HM, Nunes DP, Schroy PC 3rd, Barish MA, Clarke PD, Ferrucci JT: A comparison of virtual and conventional colonoscopy for the detection of colorectal polyps. N Engl J Med 1999;341:1496-1503.
60 Rex DK, Vining D, Kopecky KK: An initial experience with screening for colon polyps using spiral CT with and without CT colography (virtual colonoscopy). Gastrointest Endosc 1999;50:309-313.

61 Dachman AH, Kuniyoshi JK, Boyle CM, Samara Y, Hoffmann KR, Rubin DT, et al: CT colonography with three-dimensional problem solving for detection of colonic polyps. AJR Am J Roentgenol 1998;171:989-995.

62 Royster AP, Fenlon HM, Clarke PD, Nunes DP, Ferrucci JT: CT colonoscopy of colorectal neoplasms: two-dimensional and threedimensional virtual-reality techniques with colonoscopic correlation. AJR Am J Roentgenol 1997;169:1237-1242.

63 Hara AK, Johnson CD, Reed JE: Colorectal lesions: evaluation with CT colography. Radiographics 1997;17:1157-1168.

64 Mulhall BP, Veerappan GR, Jackson JL: Meta-analysis: computed tomographic colonography. Ann Intern Med 2005;142:635650.

65 Macari M, Lavelle M, Pedrosa I, Milano A Dicker M, Megibow AJ, et al: Effect of different bowel preparations on residual fluid at CT colonography. Radiology 2001;218:274277.

-66 Zalis ME, Perumpillichira J, Del Frate C, Hahn PF: CT colonography: digital subtraction bowel cleansing with mucosal reconstruction initial observations. Radiology 2003;226:911-917

67 Sosna J, Morrin MM, Kruskal JB, Lavin PT, Rosen MP, Raptopoulos V: CT colonography of colorectal polyps: a metaanalysis. AJR Am J Roentgenol 2003;181:1593-1598.

68 Halligan S, Altman DG, Taylor SA, Mallett S, Deeks JJ, Bartram CI, et al: CT colonography in the detection of colorectal polyps and cancer: systematic review, meta-analysis, and proposed minimum data set for study level reporting. Radiology 2005;237:893904.

69 Rosman AS, Korsten MA: Meta-analysis comparing CT colonography, air contrast barium enema, and colonoscopy. Am J Med 2007;120:203-210.e4.
70 Rex DK: PRO: patients with polyps smaller than $1 \mathrm{~cm}$ on computed tomographic colonography should be offered colonoscopy and polypectomy. Am J Gastroenterol 2005; 100 : 1903-1905, discussion 1907-1908.

71 Ransohoff DF: CON: immediate colonoscopy is not necessary in patients who have polyps smaller than $1 \mathrm{~cm}$ on computed tomographic colonography. Am J Gastroenterol 2005;100:1905-1907, discussion 1907-1908.

72 Pickhardt PJ: CT colonography (virtual colonoscopy) for primary colorectal screening: challenges facing clinical implementation. Abdom Imaging 2005;30:1-4.

73 Berrington de Gonzalez A, Darby S: Risk of cancer from diagnostic X-rays: estimates for the UK and 14 other countries. Lancet 2004; 363:345-351.

74 Brenner DJ, Georgsson MA: Mass screening with CT colonography: should the radiation exposure be of concern? Gastroenterology 2005;129:328-337.

75 Liedenbaum MH, Venema HW, Stoker J: Radiation dose in $\mathrm{CT}$ colonography - trends in time and differences between daily practice and screening protocols. Eur Radiol 2008;18: 2222-2230.

-76 Aschoff AJ, Ernst AS, Brambs HJ, Juchems MS: CT colonography: an update. Eur Radiol 2008; 18:429-437.

77 Levin B, Brooks D, Smith RA, Stone A: Emerging technologies in screening for colorectal cancer: CT colonography, immunochemical fecal occult blood tests, and stool screening using molecular markers. CA Cancer J Clin 2003;53:44-55.

78 Jacobs JE, Boxt LM, Desjardins B, Fishman EK, Larson PA, Schoepf J: ACR practice guideline for the performance and interpretation of cardiac computed tomography (CT). J Am Coll Radiol 2006;3:677-685.

79 Taylor SA, Laghi A, Lefere P, Halligan S, Stoker J: European Society of Gastrointestinal and Abdominal Radiology (ESGAR): consensus statement on CT colonography. Eur Radiol 2007; 17:575-579.

80 Kim DH, Pickhardt PJ, Taylor AJ: Characteristics of advanced adenomas detected at CT colonographic screening: implications for appropriate polyp size thresholds for polypectomy versus surveillance. AJR Am J Roentgenol 2007;188:940-944. 